\title{
On the estimation of the extremal index based on scaling and resampling
}

\author{
Kamal Hamidieh \\ Department of Statistics, Rice University \\ Stilian Stoev and George Michailidis \\ Department of Statistics, The University of Michigan
}

February 12, 2009

\begin{abstract}
The extremal index parameter $\theta$ characterizes the degree of local dependence in the extremes of a stationary time series and has important applications in a number of areas, such as hydrology, telecommunications, finance and environmental studies. In this study, a novel estimator for $\theta$ based on the asymptotic scaling of block-maxima and resampling is introduced. It is shown to be consistent and asymptotically normal for a large class of $m$-dependent time series. Further, a procedure for the automatic selection of its tuning parameter is developed and different types of confidence intervals that prove useful in practice proposed. The performance of the estimator is examined through simulations, which show its highly competitive behavior. Finally, the estimator is applied to three real data sets of daily crude oil prices, daily returns of the S\&P 500 stock index, and high-frequency, intra-day traded volumes of a stock. These applications demonstrate additional diagnostic features of statistical plots based on the new estimator.
\end{abstract}

Key words and phrases: Heavy tails, extremal index, resampling, permutation, bootstrap, asymptotic normality.

\section{Introduction}

Advances in computer technology have enabled the collection by research organizations and businesses of large time series data sets. These data sets are primarily characterized by the fine granularity (high frequency) of the time intervals at which the observations are collected; for example, Internet traffic is sampled at millisecond intervals, while stock trades at every second. Such time series data are characterized by the presence of long range dependence (the autocorrelation function decays at a polynomial rate) and the heavy tailed nature of the marginal distribution (see, e.g. Finkenstädt and Rootzén (2004)). In many cases, another phenomenon can be observed, namely the presence of clustering of very large or very small values (extremes) of the data (see e.g. Figure 1). For example, in Internet traces this is the result of bursty arrivals, while in data on returns of a financial asset this is primarily due to the arrival of an external market shock.

The daily log-returns of the spot price of West Texas Intermediate crude oil are shown for the period September 2006 - March 2007 in Figure 1(a) A pronounced temporal clustering of the extreme values can be seen, indicating the presence of local dependence in the extremes. Figure 1(b) also demonstrates the substantial clustering of the extremely large traded volumes in the high-frequency data set of all intra-day trading activity of the Intel stock, for example. Such clustering behavior is of interest to subject matter experts and it has important implications in practice, since it concerns large consecutive changes associated with large financial 'losses' or 'gains'. Therefore, quantifying the nature of the dependence structure as well as the duration of extreme events becomes an essential part of the understanding of these time series data. 


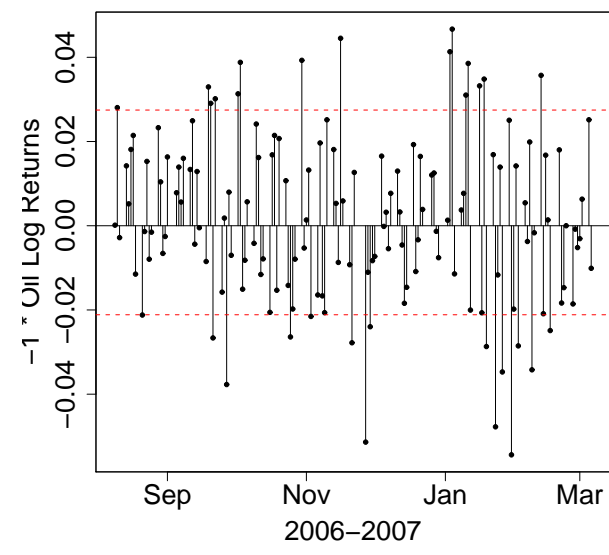

(a) Negative Log-returns of WTI Oil prices

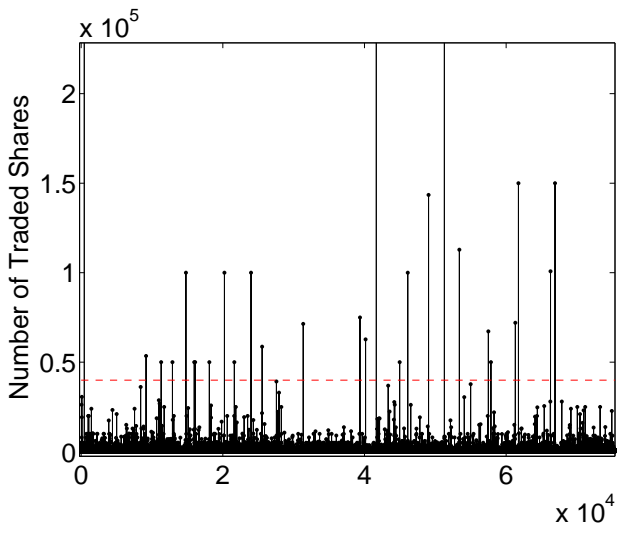

(b) High-frequency Traded Volume

Figure 1: Left plot: Negative log-returns of daily Oil prices. The upper and lower dashed lines correspond to the 0.90 and 0.10 quantiles of the data respectively. Right plot: High-frequency traded volumes (in numbers of shares per transaction) of the Intel stock in November 16, 2005. Observe the clustering of extremes, particularly evident in the extreme price drops or 'losses' (above the horizontal dotted line) for the Oil data. The trades of the Intel stock with extremely large volumes also exhibit substantial clustering.

The extremal index $\theta$ is the main parameter that describes and quantifies the clustering characteristics of the extreme values in many stationary time series. Its formal definition is given next. Let $X=\left\{X_{k}\right\}_{k \in \mathbb{Z}}$ be a strictly stationary time series. Define the following quantities

$$
M_{n}:=\max _{1 \leq k \leq n} X_{k} \quad \text { and } \quad M_{n}^{i i d}:=\max _{1 \leq k \leq n} \widetilde{X}_{k}
$$

where the $\widetilde{X}_{k}$ 's are independent and identically distributed (iid) random variables with the same distribution as the $X_{k}$ 's. Formally, the time series $X$ is said to have an extremal index $\theta$, if for some norming sequences $c_{n}>0$ and $d_{n}$, we have

$$
\mathbb{P}\left\{c_{n}^{-1}\left(M_{n}^{i i d}-d_{n}\right) \leq x\right\} \stackrel{w}{\longrightarrow} H(x) \quad \text { and } \quad \mathbb{P}\left\{c_{n}^{-1}\left(M_{n}-d_{n}\right) \leq x\right\} \stackrel{w}{\longrightarrow} H^{\theta}(x),
$$

where $H(\cdot)$ is a non-degenerate extreme value distribution (see e.g. p. 417 in Embrechts et al. (1997)).

An informal interpretation of $\theta$ is given in Leadbetter et al. (1983), namely $\theta \approx(\text { mean cluster size })^{-1}$. For example, for the crude oil log-returns discussed in Section 6 , the extremal index is estimated to be around 0.6, which means that on the average, two large size 'losses' or 'gains' are recorded in a relatively short time span. The modeling and analysis of rare events (extremes) has been an active area in probability and statistics (see e.g. Embrechts et al. (1997), Beirlant et al. (2004)). In the context of extremes, the study and the estimation of the extremal index $\theta$, plays an important role.

In this paper, we focus on the non-degenerate case when the extremal index $\theta$ is positive. Observe that in this case the same normalization and centering sequences for the partial maxima $M_{n}$ and $M_{n}^{i i d}$ above yield non-degenerate limit distributions. The extremal index takes values in the interval $[0,1]$; a value close to 0 indicates a very strong short range extremal dependence, while a value close to 1 a rather weak dependence. In fact, for iid $X_{k}$ 's, by 1.1), we have $\theta=1$. The extremal index, however, characterizes only the dependence of the extremes in the time series data and thus the data may still exhibit strong dependence, even though $\theta \approx 1$. The case of $\theta=0$ is considered to be a pathological one. 
Theoretical properties of the extremal index have been studied fairly extensively; O’Brien (1987), Hsing et al. (1988), and references therein). The problem of estimating $\theta$ has also received some attention in the literature: Hsing (1993), Smith and Weissman (1994), Weissman and Novak (1998) and Ferro and Segers (2003). Applications of the extremal index in various scientific areas include its incorporation in calculations of the Value-at-Risk measure (Longin (2000) and Klüppelberg in Finkenstädt and Rootzén (2004)), in the study of the Nasdaq and S\&P 500 indices (Galbraith and Zernov (2006)) and in the study of GARCH processes (Laurini (2004)). The estimation of the extremal index $\theta$ is an important practical problem with rapidly expanding areas of application to finance, insurance, hydrology and telecommunications, to name a few (for more details, see e.g. Embrechts et al. (1997) and Finkenstädt and Rootzén (2004)).

Most previous estimators of $\theta$ exploit its connection to the point process of exceedances. In this study, we introduce a new method for estimating $\theta$ based on the asymptotic scaling properties of block-maxima and resampling. Specifically, let $X_{1}, \ldots, X_{n}$ be a data sample from a heavy-tailed time series with positive extremal index $\theta$. The maximum values of the data calculated over blocks of size $m$, scale at a rate $m^{1 / \alpha}$, where $\alpha>0$ denotes the tail index of the marginal distribution of the data. Further, the normalized limit of the block maxima is proportional to $\theta^{1 / \alpha} \sigma$, where $\sigma:=c_{X}^{1 / \alpha}>0$ is an asymptotic scale coefficient of the $X_{k}$ 's. Thus, by examining a sequence of growing, dyadic block sizes $m=2^{j}, 1 \leq j \leq\left\lfloor\log _{2} n\right\rfloor, j \in \mathbb{N}$, and subsequently estimating the mean of logarithms of block-maxima one obtains estimating equations involving both the tail index $\alpha$ and the parameter $\theta^{1 / \alpha} \sigma$. In these equations, the scale $\sigma$ and the extremal index $\theta$ are, however, coupled. In principle, $\theta$ can be calculated by solving an appropriate nonlinear equation, but the resulting estimate proves to be too variable. Hence, we resort to resampling. Specifically, we consider either a bootstrap or a random permutation sample of the original data and then apply the previous methodology. The resampled data behaves, asymptotically, as an independent sequence with unit extremal index, that yields a second set of estimating equations of the tail index $\alpha$ and the parameter $\sigma$. By combining the resulting two estimating equations, one based on the original data and another based on the resampled data, we obtain a numerically stable estimate of $\theta$.

The resulting estimators for $\theta$ are shown to be consistent and asymptotically normal for $m$-dependent sequences, while at the same time exhibiting good mean squared error properties in finite samples. An additional advantage of resampling is that it provides a supplementary way of calculating confidence intervals for $\theta$. Resampling yields also new statistical plots, which provide further diagnostic tools for quantifying the clustering of extremes at various magnitudes. Simulation studies show that the proposed estimator is a competitive alternative to existing ones. Further, it provides new insights at the important parameter $\theta$ from the perspective of resampling, it provides new graphical tools, that can be successfully used to analyze small as well as large data sets in practice.

The remainder of the paper is organized as follows: Section 2 describes the proposed estimator. Its asymptotic properties are established in Section 3 Several methodological and algorithmic issues are discussed in Section 4, while Section 5 focuses on the evaluation of the estimator through an extensive simulation study. Three important data sets of daily Crude Oil prices, the daily returns of the S\&P 500 stock index, and the high-frequency traded volumes of the Intel stock are examined in Section 6 The proofs and some auxiliary results are given in the Appendix.

\section{The max-spectrum based estimator of $\theta$}

Let $X=\left\{X_{k}\right\}_{k \in \mathbb{Z}}$ be a positive ergodic strictly stationary sequence with heavy tailed marginals and positive extremal index $\theta>0$. Specifically, assume that $\mathbb{P}\left\{X_{k}>x\right\}=1-F(x) \sim c_{X} x^{-\alpha}$, as $x \rightarrow \infty$ for some $\alpha>0$ and $c_{X}>0$, where $a_{n} \sim b_{n}$ means $a_{n} / b_{n} \rightarrow 1$, as $n \rightarrow \infty$. The parameter $\alpha$ corresponds to the tail index of the distribution. Given a sample path $X_{1}, \ldots, X_{n}$, we define the dyadic block maxima as follows:

$$
D(j, k):=\max _{1 \leq i \leq 2^{j}} X_{2^{j}(k-1)+i} \equiv \bigvee_{i=1}^{2^{j}} X_{2^{j}(k-1)+i},
$$


where $j=1, \ldots,\left\lfloor\log _{2} n\right\rfloor, k=1, \ldots,\left\lfloor n / 2^{j}\right\rfloor$, and where $\lfloor\cdot\rfloor$ denotes the integer part function. For heavytailed $X_{k}$ 's, relation (1.1) holds with $H(x)=\exp \left\{-c_{X} x^{-\alpha}\right\}, x>0$ and normalization constants $c_{n}:=$ $n^{1 / \alpha}$ and $d_{n}:=0$. Therefore,

$$
2^{-j / \alpha} D(j, k) \stackrel{D}{\longrightarrow} \theta^{1 / \alpha} \sigma Z^{1 / \alpha}, \quad \text { as } j \rightarrow \infty, \text { for fixed } k .
$$

where $Z$ is a standard $1-$ Fréchet random variable, i.e. $\mathbb{P}\{Z \leq z\}=\exp \left(-z^{-1}\right), z>0$, and where $\sigma:=c_{X}^{1 / \alpha}$ is the asymptotic scale coefficient of the $X_{k}$ 's. Due to the nature of the Fréchet extreme value distribution, the extremal index parameter $\theta$ appears in the scale coefficient of the limit distribution of the dependent maxima. This feature will play an important role in the estimation of $\theta$ discussed below.

Next, introduce the statistics

$$
Y_{j}:=\frac{1}{n_{j}} \sum_{k=1}^{n_{j}} \log _{2}(D(j, k)) .
$$

where $n_{j}=\left\lfloor n / 2^{j}\right\rfloor$. The statistics $Y_{j}, j=1 \ldots,\left\lfloor\log _{2}(n)\right\rfloor$ will be referred to as the max-spectrum of the data, and the $j$ 's as scales. By the assumed ergodicity and provided that moments exist, for a fixed $j$, we get

$$
Y_{j} \stackrel{\text { a.s. }}{\longrightarrow} \mathbb{E} Y_{j}=j / \alpha+\mathbb{E} \log _{2}\left(2^{-j / \alpha} D(j, k)\right), \quad \text { as } n \rightarrow \infty .
$$

Assuming uniform integrability, relation 2.3 , on the other hand, implies that

$$
\mathbb{E} Y_{j} \simeq j / \alpha+\log _{2}(\sigma)+\mathbb{E} \log _{2}(Z) / \alpha+\log _{2}(\theta) / \alpha, \quad \text { as } j \rightarrow \infty,
$$

where $a_{n} \simeq b_{n}$ means $a_{n}-b_{n} \rightarrow 0$, as $n \rightarrow \infty$. This indicates the existence of a linear relationship between the statistics $Y_{j}$ and $j$ up to an error term, which becomes negligible as $n_{j}$ and $j$ grow. The slope of a linear fit of $Y_{j}$ versus $j$ yields an estimator of $1 / \alpha$ and thus $\alpha$. Although our goal is to estimate $\theta$, the estimation of the tail index $\alpha$ is an intermediate step and an integral part of our analysis.

Observe that on the other hand for iid data, we have $\theta=1$ and thus (2.6) becomes:

$$
\mathbb{E} Y_{j}^{i i d} \simeq j / \alpha+\log _{2}(\sigma)+\mathbb{E} \log _{2}(Z) / \alpha
$$

where $\left\{Y_{j}^{i i d}\right\}$ is the max-spectrum of an iid data set with the same distribution as the $X_{k}$ 's. Relations (2.6) and (2.7) suggest a method to obtain an estimate of $\theta$. Namely, resample the data, for example, by randomly drawing (with or without replacement) a sample $X_{1}^{*}, \ldots, X_{k}^{*}$ of size $k=k(n)$ from the set $\left\{X_{1}, \ldots, X_{n}\right\}$. Intuitively, this destroys the dependence structure of the data, resulting in an approximately independent sample with the same marginal distribution as the original stationary sequence.

Let $Y_{j}^{*}$ be as in (2.4) where now the $D(j, k)$ 's are based on the resampled data $X_{1}^{*}, \ldots, X_{k}^{*}$. Since for an iid sequence we have $\theta=1$, we expect the resampled sequence to have $\theta \approx 1$, whereas $\alpha$ and $\sigma$ will remain unchanged. Thus, relation 2.6 becomes

$$
\mathbb{E}\left[Y_{j}^{*}\right] \simeq j / \alpha+\log _{2}(\sigma)+\mathbb{E}\left[\log _{2}(Z)\right] / \alpha,
$$

where the term $\log _{2}(\theta) / \alpha$ is no longer present since $\log _{2}(\theta \approx 1) \approx 0$.

Thus, in view of (2.6) and (2.7), we have

$$
Y_{j}^{*} \approx j / \alpha+\log _{2}(\sigma)+\mathbb{E} \log _{2}(Z) / \alpha, \quad \text { and } \quad Y_{j} \approx j / \alpha+\log _{2}(\sigma)+\mathbb{E} \log _{2}(Z) / \alpha+\log _{2}(\theta) / \alpha .
$$

Taking the difference between the last two estimating equations, replacing $\alpha$ by its estimate $\hat{\alpha}$ based on (2.6), and solving for $\theta$ we obtain the following estimator for the extremal index:

$$
\hat{\theta}(j)=2^{-\hat{\alpha}(j)\left(Y_{j}^{*}-Y_{j}\right)} .
$$



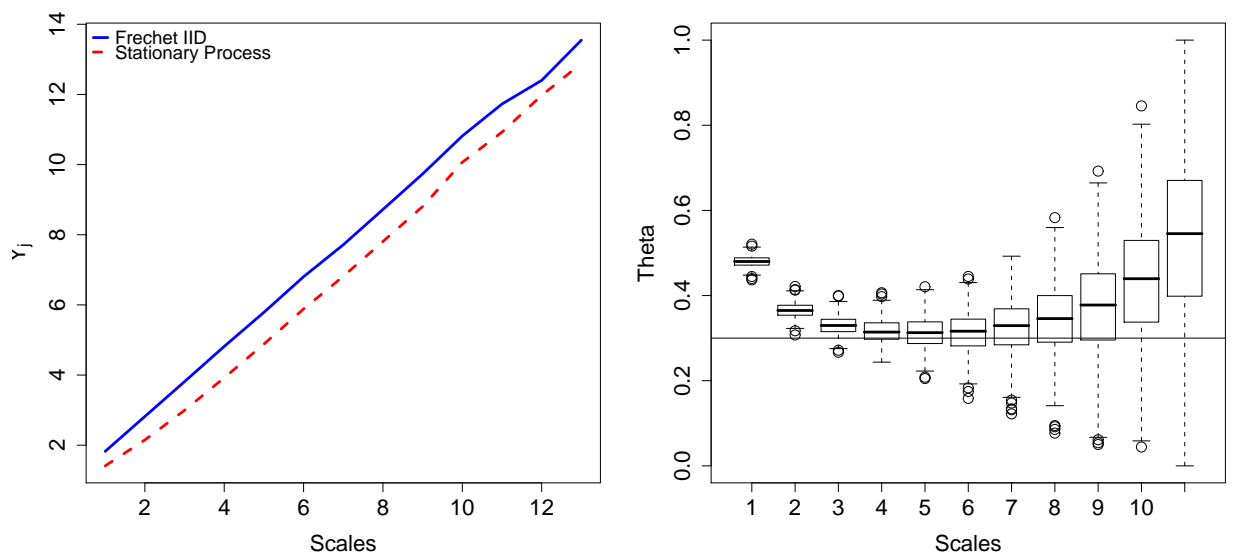

Figure 2: Left panel: The max-spectrum of $X_{n}=\max \left\{\frac{2}{3} X_{n-1}, \frac{1}{3} Z_{n}\right\}, \theta=1 / 3$, with $Z_{i}$ 's iid standard 1 -Fréchet (solid line) and the max-spectrum of iid copies of the $X_{k}$ 's (broken line). The two spectra are essentially linear with equal slopes. Right panel: boxplots of the $\hat{\theta}(j)$ 's obtained from different resampled versions of a single path of the process. The circles indicate outliers located more than 1.5 fourth-spreads away from the sample median and the horizontal line is the theoretical value of $\theta=1 / 3$.

Observe that for a single data set, one can obtain a large set of estimates $\hat{\theta}(j)$, based on different resampled versions of the data. Thus, resampling allows us to gauge the variability of the estimates as well as the range of scales $j$ where the asymptotics in (2.6) and 2.7) become applicable.

Figure 2 illustrates the main principle behind the proposed estimator. The left panel shows the combined max-spectra of a dependent sequence and an iid sample. The two max-spectra are parallel with equal slopes $\approx 1 / \alpha$, since the marginal distributions behind the two spectra are the same. The difference is in the intercept and this is where the value of $\theta$ is derived from. The right panel shows boxplots of $\hat{\theta}(j)$ estimates obtained from 200 independent resampled versions of a single path of the process on the left. Observe that the medians of the $\hat{\theta}(j)$ 's closely follow the true value $\theta=1 / 3$ over a range of scales (for more details, see Section 4 below).

Remarks:

(1) The statistics $Y_{j}$ 's in (2.4) are not only dependent in $j$, but more importantly, they have different variances in $j$ since they involve averages of $n_{j} \approx n / 2^{j}$ terms. Thus, to reduce the variance in the regression estimators of $\alpha$, it is essential to use a weighted or generalized least squares method (see e.g. Stoev et al. (2006), for more details).

(2) The proposed resampling procedure avoids the problem of estimating the scale parameter $\sigma=c_{X}^{1 / \alpha}$, however, an estimate of $\alpha$ is still needed. The algorithmic implementation of the estimators $\hat{\theta}(j)$ and other important practical issues are discussed below. The appropriate resampling sample size $k(n)$, from the perspective of asymptotics, is $o(\sqrt{n})$ (see, Section 3).

(3) The estimate $\hat{\theta}(j)$ depends on the scale $j$, as indicated. An automatic procedure for the choice of $j$ is presented in Section 4

\section{Theoretical properties}

Let $X=\left\{X_{k}\right\}_{k \in \mathbb{Z}}$ be a strictly stationary time series with marginal heavy-tailed c.d.f. $F$ and let also $M_{n}=$ $\max _{1 \leq i \leq n} X_{i} \equiv \bigvee_{i=1}^{n} X_{i}$. We then have

$$
F_{n}(x):=\mathbb{P}\left\{M_{n} \leq n^{1 / \alpha} x\right\}=\exp \left\{-c(n, x) x^{-\alpha}\right\}, \quad x \in \mathbb{R},
$$


for some function $c(n, x)>0, n \in \mathbb{N}$. As in 2.3), if the time series $X$ has a positive extremal index $\theta \in(0,1]$, then

$$
n^{-1 / \alpha} M_{n} \stackrel{D}{\longrightarrow}\left(\theta c_{X}\right)^{1 / \alpha} Z^{1 / \alpha}, \quad \text { as } n \rightarrow \infty,
$$

where $Z$ is a standard $1-$ Fréchet variable: $\mathbb{P}\{Z \leq x\}=e^{-x^{-1}}, x>0$.

Our asymptotic results rely on the moment behavior of $f\left(M_{n} / n^{1 / \alpha}\right)$, for certain deterministic functions $f$ and involve some additional technical conditions, outlined below (for more details, see the Appendix).

Condition 1. There exists $\beta>0$ and $R \in \mathbb{R}$, such that

$$
\left|c(n, x)-\theta c_{X}\right| \leq c_{1}(x) n^{-\beta}, \text { for all } x>0, \quad \text { and } \quad c_{1}(x)=\mathcal{O}\left(x^{-R}\right), x \downarrow 0,
$$

where $\theta \in(0,1]$.

Condition 2. $F_{n}(0)=0$ and for all $x>0$,

$$
c(n, x) \geq c_{2} \min \left\{1, x^{\gamma}\right\}, \text { for some } \gamma \in(0, \alpha),
$$

for all sufficiently large $n \in \mathbb{N}$, where $c_{2}>0$ does not depend on $n$.

Remarks:

(1) The conditions (3.12) and (3.13) are not very stringent. For example, let

$$
X_{k}=\max \left\{Z_{k}, Z_{k-1}, \ldots, Z_{k-m+1}\right\}, k \in \mathbb{Z},
$$

where the $Z_{k}$ 's are independent, standard $\alpha$-Fréchet. We then have

$$
\mathbb{P}\left\{M_{n} \leq n^{1 / \alpha} x\right\}=\mathbb{P}\left\{Z_{-m+1} \leq n^{1 / \alpha} x, \cdots, Z_{n} \leq n^{1 / \alpha} x\right\}=\exp \left\{-c(n, x) x^{-\alpha}\right\},
$$

where the function $c(n, x)=(n+m-1) / n=1+\mathcal{O}(1 / n)$ does not depend on $x$ and $\beta=1$, in this simple case. Conditions 1 and 2 above hold for a more general class of moving maxima processes (see Hamidieh et al. (2007)).

(2) Condition 1 and relation (3.10) imply 3.11, that is, the extremal index of the time series $X$ is precisely equal to $\theta$ in (3.12). Thus, 3.12) quantifies further the rate of the convergence in (3.11).

Description of the asymptotic regime: To obtain the consistency of statistics based on the max-spectrum $Y=\left\{Y_{j}\right\}$, we focus on the range of scales $[j(n), \ell+j(n)]$, where $\ell \in \mathbb{N}$ is fixed and where $j(n) \rightarrow \infty$, as $n \rightarrow \infty$. We then define

$$
\hat{\alpha}(j):=\left(\sum_{i=0}^{\ell} w_{i} Y_{i+j(n)}\right)^{-1},
$$

where the weights $w_{i}$ 's are fixed and such that $\sum_{i=0}^{\ell} w_{i}=0$ and $\sum_{i=0}^{\ell} i w_{i}=1$. The weights $w_{i}$ 's can be obtained, for example, either from GLS or WLS regression of $Y_{i+j(n)}$ versus $i$, for $0 \leq i \leq \ell$ (see Stoev et al. (2006), for more details).

The estimator $\hat{\theta}$ in (2.9) involves both the max-spectrum $Y$ of the dependent data and the max-spectrum $Y^{*}$ of the resampled data. Observe that

$$
\hat{\theta}(j)=2^{-\hat{\alpha}(j)\left(C^{*}(j)-C(j)\right)}, \text { where } C^{*}(j):=Y_{j}^{*}-j / \alpha \quad \text { and } \quad C(j):=Y_{j}-j / \alpha,
$$

since trivially $Y_{j}^{*}-Y_{j}=C^{*}(j)-C(j)$. We will establish the asymptotic normality of $\hat{\theta}(j)$ in three steps:

(Step 1.) We first establish rates of convergence for the quantities $\hat{\alpha}(j)$ and $C(j)$, which are based on the max-spectrum $\left\{Y_{j}\right\}$.

(Step 2.) We then show that the $C^{*}(j)$ 's are asymptotically normal (under certain conditions) in two resampling schemes: bootstrap and random permutations.

(Step 3.) We finally combine the results from Steps 1. and 2. above to establish the asymptotic normality of $\hat{\theta}(j)$.

Main results: We establish next the asymptotic normality of $\hat{\theta}(j)$ defined in (3.16), by following the three steps outlined above.

Step 1: The following result provides rates of convergence for $\hat{\alpha}(j)$ and $C(j)$. 
Proposition 3.1 Let $X_{1}, \ldots, X_{n}$ be a sample from an $m$-dependent, strictly stationary time series $X=$

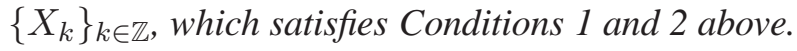

Then, for $\hat{\alpha}(j)$ and $C(j)$ in (3.15) and (3.16), we have, as $n \rightarrow \infty$

$$
\hat{\alpha}(j)=\alpha+\mathcal{O}_{P}\left(\frac{1}{2^{j(n) \min \{1, \beta\}}}\right)+\mathcal{O}_{P}\left(\frac{2^{j(n) / 2}}{n^{1 / 2}}\right), \quad \text { and } C(j)=C+\mathcal{O}_{P}\left(\frac{1}{2^{j(n) \min \{1, \beta\}}}\right)+\mathcal{O}_{P}\left(\frac{2^{j(n) / 2}}{n^{1 / 2}}\right),
$$

with $C=\log _{2}(\theta) / \alpha+\log _{2}\left(c_{X}\right) / \alpha+\mathbb{E} \log _{2}(Z) / \alpha$, where $Z$ is a standard 1 -Fréchet variable.

The proof of this result is given in the Appendix. Observe that Proposition 3.1 is valid for an arbitrary stationary $m$-dependent time series which satisfies (3.12) and (3.13). It is valid, in particular, for the simple process $\left\{X_{k}\right\}_{k \in \mathbb{Z}}$ in (3.14) and more generally for the moving maxima processes in (5.22).

Step 2: We now employ resampling to obtain an approximately independent data sample $X_{1}^{*}, \ldots, X_{k}^{*}$. Here, we consider two resampling schemes, the first based on bootstrap and the second on permutations. We then establish asymptotic normality results for the max-spectrum in both schemes. The sample $X_{1}^{*}:=$ $X_{i_{1}}, X_{2}^{*}:=X_{i_{2}}, \ldots, X_{k}^{*}:=X_{i_{k}}$ is a bootstrap sample from the data $X_{1}, \ldots, X_{n}$ if the indices $i_{1}, \ldots, i_{k}$ are drawn randomly and with replacement from the set $\{1, \ldots, n\}$. When these indices are drawn without replacement and $k \leq n$, we obtain a permutation sample. We need the following:

Lemma 3.1 Let $i_{1}, \ldots, i_{k}$ be a collection of randomly drawn indices either with replacement or without replacement from the set $\{1, \ldots, n\}$. For any fixed $m \in \mathbb{N}$, we have

$$
\mathbb{P}\left\{\min _{1 \leq j^{\prime}<j^{\prime \prime} \leq k}\left|i_{j^{\prime}}-i_{j^{\prime \prime}}\right| \geq m\right\} \geq 1-m k^{2} /(n-k) .
$$

The proof is given in the Appendix. This result implies that for $k(n)=o(\sqrt{n}), n \rightarrow \infty$, the indices $\left\{i_{j}, 1 \leq j \leq k\right\}$ are spaced by at least $m$-lags away from each other, with probability asymptotically equal to 1 , as $n \rightarrow \infty$. Therefore, if the data $X_{1}, \ldots, X_{n}$ come from an $m$-dependent time series, for the purposes of asymptotics in distribution, both the bootstrap and the permutation samples of size $k=o(\sqrt{n})$ become essentially independent, with high probability, as $n \rightarrow \infty$. This fact and Proposition 4.2 in Stoev et al. (2006), readily imply the following result.

Theorem 3.1 Let $X=\left\{X_{i}\right\}_{i \in \mathbb{Z}}$ be a strictly stationary $m$-dependent time series, which satisfies Conditions 1 and 2 above. Let $X_{1}^{*}, \ldots, X_{k}^{*}$ be either a bootstrap or a permutation sample from $X_{1}, \ldots, X_{n}$, where $k(n) \rightarrow \infty$ is such that $k(n)=o\left(n^{1 / 2}\right)$, as $n \rightarrow \infty$, and let $Y^{*}$ be its corresponding max-spectrum.

Let $j(k) \rightarrow \infty, n \rightarrow \infty$, be such that $k / 2^{j(k)(1+2 \beta)}+j(k)^{2} 2^{j(k)} / k \longrightarrow 0$, as $k \rightarrow \infty$.

Then, for $C^{*}(j)$ in (3.16), we have

$$
\sqrt{k_{j}}\left(C^{*}(j)-C^{*}\right) \stackrel{D}{\longrightarrow} \mathcal{N}\left(0, \sigma_{C^{*}}^{2}\right), \quad \text { as } n \rightarrow \infty,
$$

where $k_{j}=k(n) / 2^{j(n)}$. Here $C^{*}:=\log _{2}\left(c_{X}\right) / \alpha+\mathbb{E} \log _{2}(Z) / \alpha$, and $\sigma_{C^{*}}^{2}=\alpha^{-2} \operatorname{Var}\left(\log _{2} Z\right)$, where $Z$ is a standard $1-$ Fréchet variable.

The proof is given in the Appendix.

Step 3: The following Theorem is the main result of this Section. It combines the results of Proposition 3.1 and Theorem 3.1 to establish the asymptotic normality of $\hat{\theta}(j)$.

Theorem 3.2 Assume the conditions of Theorem 3.1 and let $\hat{\alpha}(j)$ be as in (3.15), where $Y$ is the maxspectrum of the data $X_{1}, \ldots, X_{n}$. Let also $C(j)$ and $C^{*}(j)$ be as in (3.16), where $Y^{*}$ is the max-spectrum of either a bootstrap or a permutation sample $X_{1}^{*}, \ldots, X_{k}^{*}$ of the data. 


$$
\begin{aligned}
& \text { Let } k(n)=o(\sqrt{n}), n \rightarrow \infty \text { and } j(k) \rightarrow \infty, k \rightarrow \infty \text {, be such that } \\
& \qquad k / 2^{j(k)(1+2 \min \{1, \beta\})}+j(k)^{2} 2^{j(k)} / k \rightarrow 0, \quad \text { as } k \rightarrow \infty,
\end{aligned}
$$

Then, for $\hat{\theta}(j)$ in (3.16), we have

$$
\sqrt{k_{j}}(\hat{\theta}(j)-\theta) \stackrel{D}{\longrightarrow} \mathcal{N}\left(0, \theta^{2} \pi^{2} / 6\right), \quad \text { as } n \rightarrow \infty,
$$

where $k_{j}=k(n) / 2^{j(n)}$.

The proof of this result is given in the Appendix. A few important remarks follow.

Remarks

(1) Theorem 3.2 applies, for example, to the class of moving maxima processes in (5.22), under mild assumptions on the innovations $Z_{k}$ 's (see Conditions $1^{\prime} \& 2^{\prime}$ below). It holds, for example, for Pareto, mixtures of Pareto or Fréchet innovations.

(2) Let $\delta \in(0,2 \min \{1, \beta\})$ be arbitrary and suppose that $k / 2^{j(k)(1+2 \min \{1, \beta\})} \sim k^{-\delta}, k \rightarrow \infty$. We then have $2^{j(k)} \sim k^{(1+\delta) /(1+2 \min \{1, \beta\})}, k \rightarrow \infty$ which, since $\delta<2 \min \{1, \beta\}$, implies that relation (3.19) holds. This yields the rate $k_{j} \sim k^{(2 \min \{1, \beta\}+\delta) /(1+2 \min \{1, \beta\})}$ in Theorem 3.2. Since $k=o(\sqrt{n})$ and since $\delta>0$ can be taken arbitrarily small, we can achieve rates up to $n^{\frac{\min \{1, \beta\}}{(1+2 \min \{1, \beta\})}}$. For example, if $\beta>1 / 2$ the rate of $n^{1 / 4}$ is possible while the best possible rate is $o\left(n^{1 / 3}\right)$.

\section{Implementation issues}

We present next an algorithmic implementation for the proposed estimator of $\theta$ and discuss its main features. We then propose a second algorithm for the automatic selection of scales.

In Theorem 3.2, we only consider resampled sets from the data of size $k(n)=o(\sqrt{n})$. In practice, we found that the estimators of $\theta$ continue to work well even if one considers random permutations of the entire data sample of size $k(n)=n$. Using bootstrap instead of permutation samples, results in estimates $\hat{\theta}(j)$ with larger variances and bias (for large $j$ 's), especially for small sample sizes. Thus, in the sequel, we focus on permutation based resampling and utilize the entire data set.

Algorithm 1: (estimation of $\theta$ )

1. Compute the $Y_{j}$ 's and the $\hat{\alpha}(j)$ 's as in (2.4) and (3.15) based on the original data.

2. Randomly permute (i.e. shuffle) the data, $N_{i n}$ times and collect the $N_{i n}$ statistics $Y_{j}^{*}$.

3. Find the $N_{i n}$ differences of $Y_{j}^{*}-Y_{j}$ and compute the sample mean for the positive differences only: $\Delta(j)=\operatorname{mean}\left\{Y_{j}^{*}-Y_{j}\right\}_{+}$.

4. Obtain the estimates of $\theta$ for each scale $j: \hat{\theta}(j)=\max \left\{2^{-\hat{\alpha}(j) \Delta(j)}, 1\right\}$.

5. Repeat steps 2,3 , and $4, N_{\text {out }}$ number of times and collect the $\hat{\theta}(j)$ values.

6. Produce a sequence of $\hat{\theta}(j)$ boxplots from the $N_{\text {out }}$ available values, per each scale $j$.

7. Visually inspect the boxplots of $\hat{\theta}(j)$ and select a range of scales where the medians of the boxplots stabilize. Estimate $\theta$ by using the median values from this range of scales. 

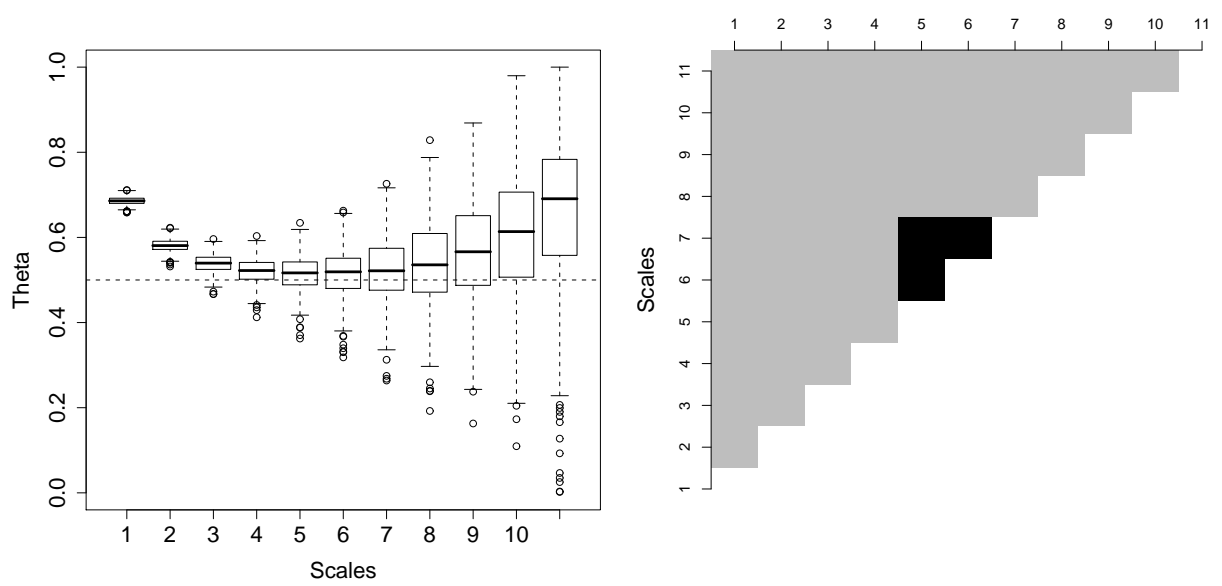

Figure 3: Estimation of $\theta$ for the process $X_{n}=\max \left\{\frac{1}{2} X_{n-1}, \frac{1}{2} Z_{n}\right\}, \theta=1 / 2, Z_{i}$ iid standard 1-Fréchet, with sample size of $\mathrm{n}=2^{13}, N_{\text {out }}=500$ and $N_{\text {in }}=25$. Left panel: Boxplots of $\hat{\theta}(j)$ 's with the last two scales omitted. Right panel: A 'heat map' visualizing the Kruskal-Wallis test for the automatic selection of scales - black corresponds to $p$-values greater than 0.05 .

In the following remarks we explain and justify the steps in the above algorithm.

\section{Discussion of Algorithm 1:}

Step 1: The estimate $\hat{\alpha}(j)$ is based on the range of scales $j, \ldots, j+\ell$, where $j+\ell=\left\lfloor\log _{2}(n)\right\rfloor-1$ is chosen to be the second largest available scale in the data. In practice, we discard the highest scale since it involves an average of at most two block-maxima. We recommend using either generalized least squares with the asymptotic covariance matrix for the max-spectrum given in Stoev et al. (2006) or weighted least squares which account for the fact that $\operatorname{Var}\left(Y_{j}\right) \propto 1 / n_{j} \propto 2^{j}$. Both approaches are comparable and considerably better than ordinary least squares regression, which should not be used.

Steps $2 \& 3$ : We introduce an inner loop with $N_{i n}$ iterations to reduce the variability of $Y_{j}^{*}-Y_{j}$. This considerably improves the variance of the $\theta$ estimates. On step 3, we average only the positive differences $Y_{j}^{*}-Y_{j}$ since by relations (2.6) and (2.8), we have $\mathbb{E} Y_{j}^{*} \geq \mathbb{E} Y_{j}$. Our experiments indicate that replacing the "mean" by "median" in step 3 yields similar results.

Step 4: As in Ferro and Segers (2003), we take the minimum of the calculated estimate and 1 to ensure that $\hat{\theta}(j) \in[0,1]$.

Step 5: This step yields a sample of $N_{\text {out }}$ estimates of $\theta$ for each scale $j$. The practical choice of the parameters $N_{\text {out }}$ and $N_{\text {in }}$ is discussed in Section 5

Step 6: In practice, the estimation of $\theta$ requires selecting the range of scales, where the best bias/variance trade-off is achieved. Estimating $\theta$ over the larger scales $j$ (larger block sizes) involves lower bias, but leads to larger variance as the number of block-maxima is reduced. At lower scales $j$ (smaller block sizes) the bias grows but the variance is reduced (see Figure 3). In general, reliable estimates of $\theta$ can be obtained from the middle range of scales. The choice of the scales $j$ is addressed in the sequel.

Figure 3(left panel) illustrates the above algorithm over a simulated process with known extremal index $\theta=1 / 2$. A stable range of scales 4 to 7 can be observed. In practice, we recommend taking the median of the sample of the pooled $N_{\text {out }}$ estimates $\hat{\theta}(j)$ from each one of the scales $j$ in the stable range. In this case we obtained a point estimate of 0.52 . One can also obtain an empirical $95 \%$ confidence interval, based on 0.025 -th and 0.975 -th empirical quantiles of the pooled $\hat{\theta}(j)$ values to obtain $(0.40,0.62)$ (see also relations (5.23) and (5.24) below). 
The selection of the stable range of scales $j$ in Step 6 of Algorithm 1 is subjective. We propose next an automated procedure for selecting the range of scales, based on the Kruskal-Wallis test.

Algorithm 2: (automatic selection of scales)

1. For every given range $j_{1} \leq j \leq j_{2}, j_{1}<j_{2}$ of possible consecutive scales in the data, perform a Kruskal-Wallis test for equality of the medians, based on the samples of $N_{\text {out }}$ values of $\hat{\theta}(j)$.

2. Consider the array of $p$-values: $p\left(j_{1}, j_{2}\right)$ resulting from the tests in Step 1. Declare the medians over the range $\left[j_{1}, j_{2}\right]$ 'statistically different' if $p$ is less than a prescribed significance threshold.

3. Produce a pooled estimate of $\theta$ based on the longest scale range where the medians are 'statistically equal'.

4. If there are ties in Step 3, pick the range starting at the lowest scale. If all medians are 'statistically different', pick the middle scale and follow up by a visual inspection of the results.

The proposed automatic scale selection procedure is evaluated in Section 5, One possible method to visualize the results of this analysis is to construct a 'heat map' of the p-values for the Kruskal-Wallis tests - see Figure 3 (right panel). The axes correspond to scales $j_{1}$ and $j_{2}$; the regions in black indicate ranges of scales $\left[j_{1}, j_{2}\right]$ with $p$-values greater than 0.05 . This heat map shows that the medians over the scale range $\left[j_{1}, j_{2}\right]=[5,7]$ are 'statistically equal' at a level of $5 \%$. A point estimate based on the pooled values from scales 5 to 7 is 0.52 with an empirical $95 \%$ confidence interval of $(0.39,0.63)$.

\section{Performance evaluation}

We present next the results of a simulation study and comment on the performance of the max-spectrum, the Ferro-Segers (Ferro and Segers (2003)) and the runs (O'Brien (1987)) estimators for the extremal index. We briefly summarize these two competing estimators next:

The first estimator is based on the characterization of the extremal index given by O'Brien (1987). In this characterization, $\theta$ is expressed as the limiting probability that an exceedance is followed by a run of observations below a high threshold $u_{n}$ :

$$
\theta=\lim _{n \rightarrow \infty} P\left\{\bigvee_{j=2}^{r_{n}} X_{j} \leq u_{n} \mid X_{1}>u_{n}\right\},
$$

where $r_{n}=o(n)$ is the length of runs of values of the process falling below the threshold given that an exceedance has occurred. This characterization motivates the definition of the runs estimator for a fixed high threshold $u$ and a specified runs length $r$ :

$$
\hat{\theta}_{\text {runs }}=\frac{\sum_{j=1}^{n-r} \mathbf{I}\left(X_{j} \geq u \geq \bigvee_{i=j+1}^{j+r} X_{i}\right)}{\sum_{j=1}^{n-r} \mathbf{I}\left(X_{j}>u\right)} .
$$

The runs estimator is asymptotically normal and consistent. See Weissman and Novak (1998) and references therein for additional information.

The second estimator is due to Ferro and Segers (2003). An interesting aspect of this estimator is that it does not require an auxiliary parameter (run length in the case of the runs estimator). However, one still has to choose the threshold. Using a point process approach, Ferro and Segers (2003) show that the interexceedance times - time differences between successive values above a threshold - of the extreme values 
normalized by $\bar{F}\left(u_{n}\right)$ converge in distribution to a random variable $T_{\theta}$ with a mass of $1-\theta$ at $t=0$ and an exponential distribution with rate equal to $\theta$ on $t>0$. Using a moment estimator, they first obtain:

$$
\hat{\theta}_{1}=\frac{2\left(\sum_{i=1}^{N-1} T_{i}\right)^{2}}{(N-1)\left(\sum_{i=1}^{N-1} T_{i}^{2}\right)},
$$

where $\left\{T_{i}\right\}$ are the inter-exceedance times and $N$ is the number of exceedances of a fixed high threshold $u$. A bias corrected version gives,

$$
\hat{\theta}_{2}=\frac{2\left(\sum_{i=1}^{N-1}\left(T_{i}-1\right)\right)^{2}}{(N-1)\left(\sum_{i=1}^{N-1}\left(T_{i}-1\right)\left(T_{i}-2\right)\right)} .
$$

To obtain the final form of the estimator, a further adjustment is made to ensure that the values of the estimator lie between 0 and 1 :

$$
\hat{\theta}_{F / S}= \begin{cases}1 \wedge \hat{\theta}_{1} & \text { if } \max \left\{T_{i}: 1 \leq i<N-1\right\} \leq 2 \\ 1 \wedge \hat{\theta}_{2} & \text { if } \max \left\{T_{i}: 1 \leq i<N-1\right\}>2\end{cases}
$$

The Ferro-Segers estimator is consistent for $m$-dependent strictly stationary sequences.

Next, we discuss three types of processes, used in the simulation study, for which the extremal index is given in closed form.

- The max-autoregressive (armax) process of order one is defined as:

$$
X_{n}=\max \left\{b X_{n-1},(1-b) Z_{n}\right\}, \quad \text { where } 0 \leq b<1,
$$

and where $\left\{Z_{n}\right\}_{n \in \mathbb{Z}}$ is an iid sequence of standard $\alpha$-Fréchet random variables. For such processes $\theta=$ $1-b^{\alpha}$ can take any value in the interval $(0,1]$ (see e.g. Beirlant et al. (2004) for additional information).

- The linear process $\left\{Y_{n}\right\}, n \in \mathbb{Z}$ is defined as:

$$
Y_{n}=\sum_{j \in \mathbb{Z}} \psi_{j} Z_{n-j}, n \in \mathbb{Z}, \quad \text { where } \sum_{j \in \mathbb{Z}}\left|\psi_{j}\right|^{\delta}<\infty, \quad \text { for some } 0<\delta<\min \{1, \alpha\} .
$$

Here $\left\{Z_{n}\right\}_{n \in \mathbb{Z}}$ is an iid sequence of heavy-tailed innovations with exponent $\alpha>0$. When the $Z_{n}$ 's are symmetric, we have $\theta=\left(\psi_{+}^{\alpha}+\psi_{-}^{\alpha}\right) /\|\psi\|_{\alpha}^{\alpha}$, where $\psi_{+}=\max _{j}\left(\psi_{j} \vee 0\right), \psi_{-}=\max _{j}\left(-\psi_{j} \vee 0\right)$, and $\|\psi\|_{\alpha}^{\alpha}=\sum_{j \in \mathbb{Z}}\left|\psi_{j}\right|^{\alpha}$ (see, e.g. Corollary 5.5.3 in Embrechts et al. (1997)). We will use iid t-distributed innovations $Z_{n}$ 's where the degrees of freedom parameter is also equal to the tail index $\alpha$.

- The moving maxima process $X=\left\{X_{k}\right\}_{k \in \mathbb{Z}}$ is defined as:

$$
X_{k}:=\max _{1 \leq i \leq m} a_{i} Z_{k-i+1}, \quad k \in \mathbb{Z},
$$

with some coefficients $a_{i}>0, i=1, \ldots, m$, and $m \geq 1$, where the $Z_{k}$ 's are iid, positive heavy-tailed random variables with tail exponent $\alpha$. The extremal index $\theta$ of $X$ is: $\theta=\max _{1 \leq i \leq m} a_{i}^{\alpha} / \sum_{i=1}^{m} a_{i}^{\alpha}$.

Simulation setup: For brevity, we present selected results for the processes under consideration that demonstrate best the behavior of the various estimators.

$\circ X_{n}=\max \left\{b X_{n-1},(1-b) Z_{n}\right\}$, with $Z_{i}$ iid standard 1-Fréchet.

$\circ Y_{n}=0.50 Z_{n}+0.20 Z_{n-1}+0.10 Z_{n-2}$, with $Z_{i}$ iid t-distributed with $\alpha$ degrees of freedom.

$\circ W_{n}=\max \left\{0.80 Z_{n}, 0.20 Z_{n-1}, 0.40 Z_{n-2}\right\}$, with $Z_{i}$ iid Pareto with tail index $\alpha$.

- Parameters: For the armax processes, we fix the tail index at $\alpha=1$ and vary the coefficient $b$ to obtain a range of $\theta$ values. The coefficients of the linear and moving maxima processes are fixed (as indicated above), and the values of $\alpha$ for the $Z_{k}$ 's are varied to obtain a range of $\theta$ values. For all processes, other choices of the parameters produced analogous results. For each type of process, 500 independent sample paths were generated of length $2^{13}=8192$ for the armax and moving max processes and $2^{14}=16384$ for the linear 
processes. For each generated sample path, the Ferro-Segers, the runs 1, 5, and 9 at each selected threshold were computed. The proposed max-spectrum based estimator was computed using both GLS and WLS and setting $N_{i n}=25$. The threshold (Ferro-Segers and runs estimators) and the scale (proposed estimator) parameters achieving the best Root-Mean-Square-Error (RMSE) are reported in Tables 1-3.

The results demonstrate that the proposed max-spectrum estimator exhibits a good overall performance in terms of RMSE and in many settings it outperforms the Ferro-Segers estimator. The GLS and WLS variants produce similar results. The runs estimator performs exceptionally well for the armax process, if the 'correct' run-length parameter is specified. However, it is quite sensitive to the type of process and to the choice of the run-length parameter employed. The max-spectrum and Ferro-Segers estimators are significantly more robust than the runs estimator to the choice of the model.

\begin{tabular}{|c|c|c|c|c|c|c|c|}
\hline$\theta$ & $\alpha$ & $G L S$ & $W L S$ & $F / S$ & Runs -1 & Runs -5 & Runs -9 \\
\hline \hline 0.10 & 1.00 & 0.0189 & 0.0197 & 0.0140 & $\mathbf{0 . 0 1 0 9}$ & 0.0127 & 0.0137 \\
\hline 0.20 & 1.00 & 0.0226 & 0.0256 & 0.0206 & $\mathbf{0 . 0 1 6 4}$ & 0.0218 & 0.0247 \\
\hline 0.30 & 1.00 & 0.0325 & 0.0291 & 0.0272 & $\mathbf{0 . 0 2 2 3}$ & 0.0298 & 0.0343 \\
\hline 0.40 & 1.00 & 0.0334 & 0.0290 & 0.0306 & $\mathbf{0 . 0 2 7 2}$ & 0.0381 & 0.0440 \\
\hline 0.50 & 1.00 & 0.0335 & 0.0308 & 0.0316 & $\mathbf{0 . 0 3 0 2}$ & 0.0436 & 0.0520 \\
\hline 0.60 & 1.00 & 0.0350 & $\mathbf{0 . 0 3 1 0}$ & 0.0326 & 0.0316 & 0.0485 & 0.0569 \\
\hline 0.70 & 1.00 & 0.0323 & $\mathbf{0 . 0 2 8 5}$ & 0.0348 & 0.0327 & 0.0493 & 0.0584 \\
\hline 0.80 & 1.00 & 0.0274 & $\mathbf{0 . 0 2 4 3}$ & 0.0365 & 0.0323 & 0.0508 & 0.0638 \\
\hline 0.90 & 1.00 & 0.0212 & $\mathbf{0 . 0 2 0 6}$ & 0.0363 & 0.0284 & 0.0506 & 0.0621 \\
\hline
\end{tabular}

Table 1: RMSE values for $X_{n}=\max \left\{b X_{n-1},(1-b) Z_{n}\right\}$, with $Z_{i}$ iid standard $1-$ Fréchet. The first column contains the $\theta$ values. The last 6 columns contain the best RMSE values for the max-spectrum estimates via GLS, WLS, and the competitors. The sample sizes were fixed at $2^{13}$, with $N_{\text {out }}=500$, and $N_{\text {in }}=25$.

\begin{tabular}{|c|c|c|c|c|c|c|c|}
\hline$\theta$ & $\alpha$ & $G L S$ & $W L S$ & $F / S$ & Runs -1 & Runs -5 & Runs -9 \\
\hline \hline 0.36 & 0.10 & 0.0226 & 0.0291 & 0.0172 & $\mathbf{0 . 0 1 0 0}$ & 0.0155 & 0.0198 \\
\hline 0.48 & 0.50 & 0.0262 & 0.0299 & 0.0204 & $\mathbf{0 . 0 1 8 1}$ & 0.0322 & 0.0373 \\
\hline 0.63 & 1.00 & 0.0328 & 0.0315 & $\mathbf{0 . 0 2 3 5}$ & 0.0265 & 0.0441 & 0.0509 \\
\hline 0.74 & 1.50 & 0.0226 & $\mathbf{0 . 0 2 0 3}$ & 0.0404 & 0.0333 & 0.0509 & 0.0611 \\
\hline 0.83 & 2.00 & $\mathbf{0 . 0 1 4 7}$ & 0.0238 & 0.0598 & 0.0412 & 0.0576 & 0.0667 \\
\hline 0.89 & 2.50 & 0.0032 & 0.0162 & 0.0007 & 0.0003 & $\mathbf{0 . 0 0 0 0}$ & 0.0007 \\
\hline 0.93 & 3.00 & 0.0013 & 0.0004 & $\mathbf{0 . 0 0 0 2}$ & 0.0043 & 0.0043 & 0.0043 \\
\hline
\end{tabular}

Table 2: RMSE values for $Y_{n}=0.50 Z_{n}+0.20 Z_{n-1}+0.10 Z_{n-2}$, with $Z_{i}$ iid t-distributed. The first column contains the $\theta$ values. The tail index values are in the second column. The last 6 columns contain the best RMSE values for the max-spectrum estimates via GLS, WLS, and the competitors. The sample sizes were fixed at $2^{14}$, with $N_{\text {out }}=500$, and $N_{\text {in }}=25$.

Figure 4 shows boxplots of 500 independent realizations of the WLS variant of the max-spectrum estimator, computed for a linear process with $\theta=0.625$. The boxplots for the WLS (GLS boxplots were very similar) method and the median of the estimates of the Ferro-Segers and the runs estimators per threshold are shown. The runs estimator is quite sensitive to the choice of the run-length and exhibits systematic bias. The Ferro-Segers and max-spectrum estimators are more robust and do not exhibit such strong bias, a fact observed in numerous other experimental settings.

On the choice of $N_{\text {in }}$ and $N_{\text {out }}$ : The choice of the resampling parameters $N_{\text {in }}$ and $N_{\text {out }}$ in Step 5 of Algorithm 1 involves an intricate bias-variance trade off. Our experience with various sample sizes $n$ and values for $N_{\text {in }}$ and $N_{\text {out }}$ shows that larger values for $N_{\text {in }}$ lead to smaller variances but larger bias. Extremely large values of $N_{\text {out }}$ may not yield a good resampling approximation of the distribution of the $\hat{\theta}(j)$ 's. In real data and/or for smaller samples (e.g. up to several thousands), we recommend using $N_{i n}=1$ and 


\begin{tabular}{|c|c|c|c|c|c|c|c|}
\hline$\theta$ & $\alpha$ & $G L S$ & $W L S$ & $F / S$ & Runs -1 & Runs -5 & Runs -9 \\
\hline \hline 0.36 & 0.10 & 0.0212 & 0.0287 & 0.0212 & $\mathbf{0 . 0 0 8 5}$ & 0.0143 & 0.0181 \\
\hline 0.45 & 0.50 & $\mathbf{0 . 0 2 4 4}$ & 0.0311 & 0.0256 & 0.0557 & 0.0274 & 0.0334 \\
\hline 0.57 & 1.00 & $\mathbf{0 . 0 3 1 5}$ & 0.0325 & 0.0329 & 0.0867 & 0.0400 & 0.0474 \\
\hline 0.68 & 1.50 & 0.0353 & $\mathbf{0 . 0 3 4 0}$ & 0.0350 & 0.0844 & 0.0471 & 0.0560 \\
\hline 0.76 & 2.00 & 0.0348 & $\mathbf{0 . 0 3 2 8}$ & 0.0365 & 0.0606 & 0.0482 & 0.0571 \\
\hline 0.83 & 2.50 & $\mathbf{0 . 0 3 2 0}$ & 0.0323 & 0.0378 & 0.0324 & 0.0527 & 0.0625 \\
\hline 0.88 & 3.00 & 0.0301 & 0.0297 & 0.0400 & $\mathbf{0 . 0 1 2 4}$ & 0.0501 & 0.0594 \\
\hline
\end{tabular}

Table 3: RMSE values for $W_{n}=\max \left\{0.80 Z_{n}, 0.20 Z_{n-1}, 0.40 Z_{n-2}\right\}$, with $Z_{i}$ iid Pareto. The first column contains the $\theta$ values. The tail index values are in the second column. The last 6 columns contain the best RMSE values for the max-spectrum estimates via GLS, WLS, and the competitors. The sample sizes were fixed at $2^{13}$, with $N_{\text {out }}=500$, and $N_{\text {in }}=25$.
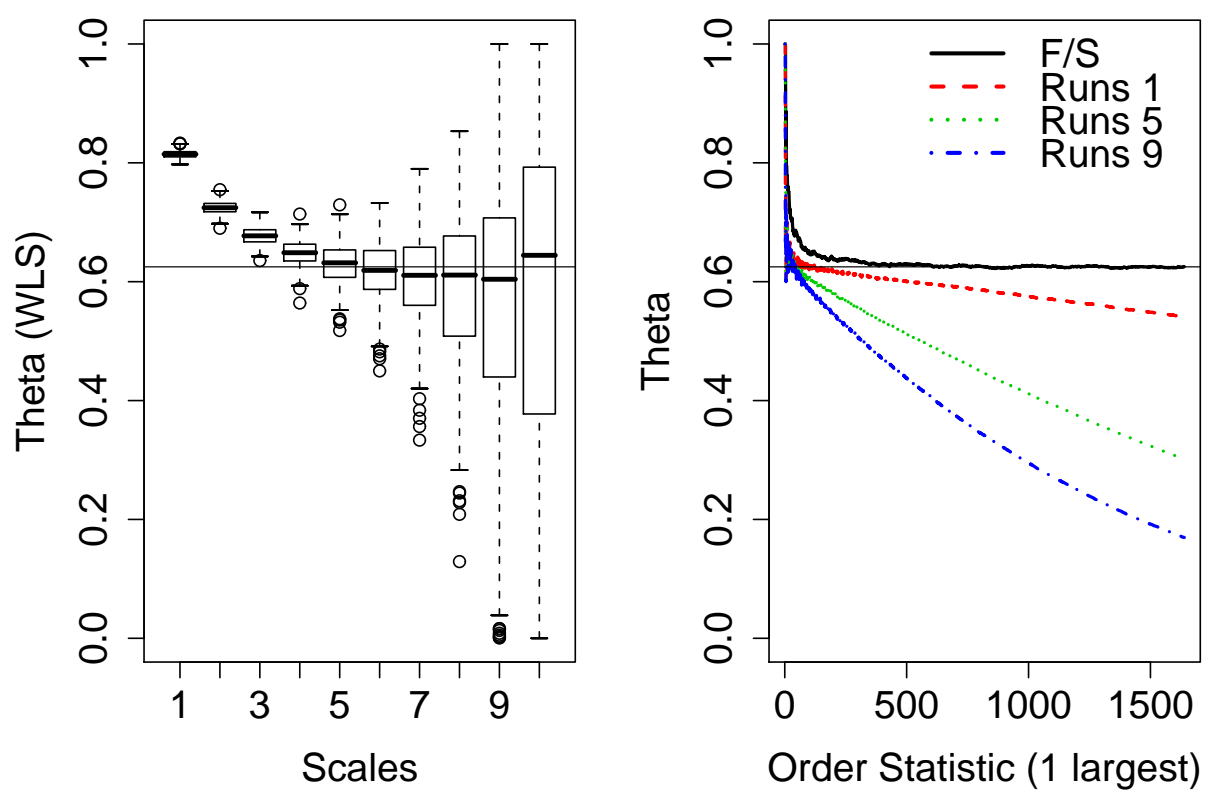

Figure 4: WLS simulation results for $Y_{n}=0.50 Z_{n}+0.20 Z_{n-1}+0.10 Z_{n-2}, \theta=0.625, Z_{i}$ iid t-distributed with $d f=\alpha=1.00$, and a sample size of $2^{14}$, with $N_{\text {out }}=500$ and $N_{\text {in }}=25$. Left panel: Boxplots of max-spectrum $\hat{\theta}$. Right panel: $\hat{\theta}$ obtained form the runs and Ferro-Segers estimators. In both plots, the solid horizontal line corresponds to $\theta=0.625$.

$N_{\text {out }}=200$, for example. Using $N_{\text {in }}=1$ yields slightly larger variances, leading to wider confidence intervals, but prevents missing the 'true value' due to elevated bias. For moderate and large samples, and if computation time may be of a lesser concern, we recommend using $N_{i n}>1$. The choice of $N_{\text {in }}>1$, reduces the variance of the estimators, and as long as the value $N_{\text {in }} \times N_{\text {out }}$ is not too large, relative to the available sample size, this does not lead to elevated bias.

Automatic selection of scales: We illustrate next the performance of the automatic selection procedure, introduced in Section 4 We use a subset of the armax, linear and moving maxima processes, described in the simulation setup above. As before, for each process, we generate 500 independent realizations, of length $2^{13}=8192$ for the armax $(\mathrm{AM})$ and moving maxima $(\mathrm{MM})$ processes and $2^{14}=16384$ for the linear 


\begin{tabular}{|c|c|c||c|c|c||c|c|c|}
\hline \multicolumn{4}{|c|}{} & \multicolumn{3}{c|}{ Best Scale } & \multicolumn{3}{c|}{ Automatic Selection } \\
\hline \hline Process & $\theta$ & $\alpha$ & RMSE & Median & SD & RMSE & Median & $S D$ \\
\hline \hline$A M$ & 0.20 & 1.00 & 0.0252 & 0.22 & 0.0195 & 0.0439 & 0.22 & 0.0404 \\
\hline$A M$ & 0.50 & 1.00 & 0.0313 & 0.52 & 0.0268 & 0.0748 & 0.52 & 0.0713 \\
\hline$A M$ & 0.80 & 1.00 & 0.0257 & 0.81 & 0.0221 & 0.0717 & 0.81 & 0.0702 \\
\hline \hline$L P$ & 0.48 & 0.50 & 0.0303 & 0.49 & 0.0301 & 0.0672 & 0.48 & 0.0670 \\
\hline$L P$ & 0.74 & 1.50 & 0.0200 & 0.76 & 0.0154 & 0.0635 & 0.74 & 0.0631 \\
\hline$L P$ & 0.89 & 2.50 & 0.0230 & 0.87 & 0.0090 & 0.0738 & 0.84 & 0.0620 \\
\hline \hline$M M$ & 0.45 & 0.50 & 0.0324 & 0.47 & 0.0271 & 0.0513 & 0.47 & 0.0493 \\
\hline$M M$ & 0.68 & 1.50 & 0.0336 & 0.69 & 0.0276 & 0.0666 & 0.69 & 0.0638 \\
\hline$M M$ & 0.83 & 2.50 & 0.0337 & 0.85 & 0.0226 & 0.0700 & 0.84 & 0.0686 \\
\hline
\end{tabular}

Table 4: Best RMSE values versus the RMSE from the automatic scale selection procedure.

processes (LP). We now use $N_{\text {out }}=200$ and $N_{\text {in }}=1$ and thus we obtain 200 dependent estimates of $\theta$ per scale $j$, for each sample path. We apply the automatic selection procedure based on the Kruskal-Wallis test (at a level of $5 \%$ ) for each set of 200 resampled $\theta$ estimates. We thus obtain a single $\theta$ estimate per simulated path.

This procedure is repeated for each independent realization and RMSE values are computed based on the obtained $\theta$ estimates from the automatic procedure. We report the best RMSE value (lowest RMSE value among scales), the median and the standard deviation of the estimates based on the automatic procedure and the same values corresponding to the scale at which the best RMSE value was obtained (as in Tables 13 ).

Table4/indicates that the automatic selection procedure performs very well in terms of bias (as compared to the best-RMSE scale). The RMSE values for the automatic selection method are larger than the bestscale-RMSE values. This is due to the larger variance as seen from the reported standard deviations. Such a behavior is to be expected since the automatic selection procedure does not involve any knowledge of the true value of $\theta$. In practice, since $\theta$ is unknown, one cannot identify the best scale $j$ and hence one cannot achieve the best-RMSE. In such a setting the automatic selection procedure appears to perform well, by producing estimates with low bias and paying a small price in higher variability.

Confidence Intervals: The following variants of confidence intervals were constructed and compared. The first, based on asymptotic normality (see Theorem 3.2), is given by

$$
\hat{\theta}(j) \pm z_{(1-q) / 2} \hat{\theta}(j) \pi \sqrt{1 / 6 n_{j}},
$$

where $z_{(1-q) / 2}$ is a $(1-q) / 2$-th quantile of the standard normal distribution and $n$ and $n_{j}=\left\lfloor n / 2^{j}\right\rfloor$ are the total sample size and the number of block-maxima involved in the calculation of the $Y_{j}$ statistic, respectively. Table 5 displays coverage probabilities for nominal levels .05 and .10 for scales $j$ between 4 and 8 , where the $\hat{\theta}(j)$ estimates typically stabilize. These results are based on 500 independent realizations for each process.

The second type of confidence intervals are based on resampled versions of a single sample path of the data. The computed $\theta$ estimates are pooled across a range of scales with reasonable estimates, and then take the appropriate empirical quantiles:

$$
\left(\hat{\theta}\left(j_{1}, j_{2}\right)_{\left(\frac{1-q}{2}\right)}, \hat{\theta}\left(j_{1}, j_{2}\right)_{\left(\frac{1+q}{2}\right)}\right),
$$

where $\hat{\theta}\left(j_{1}, j_{2}\right)_{(\tau)}$ represents the empirical $\tau$-th quantile of the pooled $\hat{\theta}(j)$ values across scales $j_{1} \leq j \leq j_{2}$. The coverage probabilities based on (5.24) are reported in Table 6

Tables 5-6 show coverage probabilities for the middle range of scales. The confidence intervals based on the asymptotic approximation tend to over-cover the parameter $\theta$, as compared to the nominal level. On the other hand, the resampled based confidence intervals tend to undercover $\theta$, on the average. Further, experience shows that for lower scales, the coverage probabilities suffer substantially due to bias; however, as $j$ 


\begin{tabular}{|c|c|c||c|c|c|c|c||c|c|c|c|c|}
\hline \multicolumn{9}{|c|}{} & \multicolumn{5}{|c|}{$90 \%$ - Scales } & \multicolumn{5}{|c|}{$95 \%$ - Scales } \\
\hline \hline Process & $\theta$ & $\alpha$ & 4 & 5 & 6 & 7 & 8 & 4 & 5 & 6 & 7 & 8 \\
\hline \hline$A M$ & 0.20 & 1.00 & 36 & 72 & 82 & 85 & 89 & 48 & 84 & 90 & 93 & 96 \\
\hline$A M$ & 0.50 & 1.00 & 88 & 96 & 96 & 96 & 96 & 94 & 99 & 98 & 99 & 99 \\
\hline$A M$ & 0.80 & 1.00 & 99 & 99 & 99 & 99 & 98 & 100 & 100 & 100 & 100 & 99 \\
\hline \hline$L P$ & 0.48 & 0.50 & 56 & 81 & 80 & 72 & 65 & 68 & 88 & 85 & 78 & 70 \\
\hline$L P$ & 0.74 & 1.50 & 94 & 90 & 88 & 84 & 79 & 98 & 95 & 93 & 89 & 83 \\
\hline$L P$ & 0.89 & 2.50 & 49 & 80 & 90 & 89 & 86 & 62 & 87 & 93 & 93 & 89 \\
\hline \hline$M M$ & 0.45 & 0.50 & 68 & 95 & 99 & 99 & 99 & 82 & 98 & 100 & 100 & 100 \\
\hline$M M$ & 0.68 & 1.50 & 93 & 99 & 99 & 100 & 100 & 98 & 100 & 100 & 100 & 100 \\
\hline$M M$ & 0.83 & 2.50 & 99 & 99 & 100 & 100 & 99 & 100 & 100 & 100 & 100 & 99 \\
\hline
\end{tabular}

Table 5: Coverage probabilities for a selected set of processes using equation (5.23).

\begin{tabular}{|c|c|c||c|c|c|c|c||c|c|c|c|c|}
\hline \multicolumn{9}{|c|}{} & \multicolumn{5}{|c|}{$90 \%$ - Scales } & \multicolumn{5}{|c|}{$95 \%$ - Scales } \\
\hline \hline Process & $\theta$ & $\alpha$ & 4 & 5 & 6 & 7 & 8 & 4 & 5 & 6 & 7 & 8 \\
\hline \hline$A M$ & 0.20 & 1.00 & 10 & 33 & 37 & 34 & 31 & 13 & 38 & 43 & 40 & 34 \\
\hline$A M$ & 0.50 & 1.00 & 34 & 58 & 62 & 61 & 61 & 40 & 66 & 69 & 67 & 68 \\
\hline$A M$ & 0.80 & 1.00 & 75 & 79 & 79 & 80 & 81 & 83 & 85 & 86 & 88 & 87 \\
\hline \hline$L P$ & 0.48 & 0.50 & 31 & 61 & 58 & 56 & 53 & 36 & 69 & 66 & 64 & 60 \\
\hline$L P$ & 0.74 & 1.50 & 79 & 75 & 75 & 71 & 74 & 86 & 82 & 82 & 80 & 80 \\
\hline$L P$ & 0.89 & 2.50 & 20 & 57 & 75 & 82 & 83 & 28 & 65 & 82 & 90 & 90 \\
\hline \hline$M M$ & 0.45 & 0.50 & 17 & 55 & 68 & 74 & 79 & 20 & 63 & 75 & 81 & 87 \\
\hline$M M$ & 0.68 & 1.50 & 31 & 67 & 78 & 81 & 83 & 37 & 79 & 86 & 88 & 90 \\
\hline$M M$ & 0.83 & 2.50 & 60 & 81 & 84 & 85 & 84 & 70 & 88 & 92 & 91 & 89 \\
\hline
\end{tabular}

Table 6: Coverage probabilities for a selected set of processes using equation (5.24).

increases the results rapidly improve. These results indicate that the information from the two types of confidence intervals, combined, provides useful ball-park estimates for accurate confidence interval estimates for $\theta$. The difficult problem of obtaining accurate confidence intervals for $\theta$ which work well in practice will be the focus of future work.

\section{Applications}

Crude Oil Data: The daily log returns of West Texas Intermediate (WTI) crude oil prices from January 2, 1986 to March 6, 2007 (5744 observations) are analyzed and the extremal index estimated. Note that the daily log returns (referred as returns henceforth) are approximately equal to the daily percentage changes in the price. WTI represents a benchmark against which all oil bound for the US is priced at and hence its market is deep and liquid. The data were obtained from Energy Information Administration (see http://www.eia.doe.gov/). For a useful reference on oil markets see Geman (2005).

Figure 5 shows a plot of the data and the corresponding returns. The return series appears to be approximately stationary, with the exception of a few instances, the result of events of major economic impact. In the top panel, the run up of the oil prices before the first Persian Gulf war can be seen, together with its subsequent rapid drop once it became apparent that the coalition forces would prevail. A similar pattern is observed at the onset of the recent Iraq war. The run up in oil prices over the course of the last three years, accentuated due to sustained demand and growth is also evident in the plot, together with their steep drop starting in mid-July 2008.

Analysis of the tail behavior of the data by examining the max-spectrum and Hill estimators indicate a value of $\alpha \approx 3$ and 2.5 for the right and left tails, respectively. We study separately the time series of positive (right tail of the distribution) and negative (left tail) returns. This is motivated by the empirical fact that positive and negative returns exhibit different behavior. 

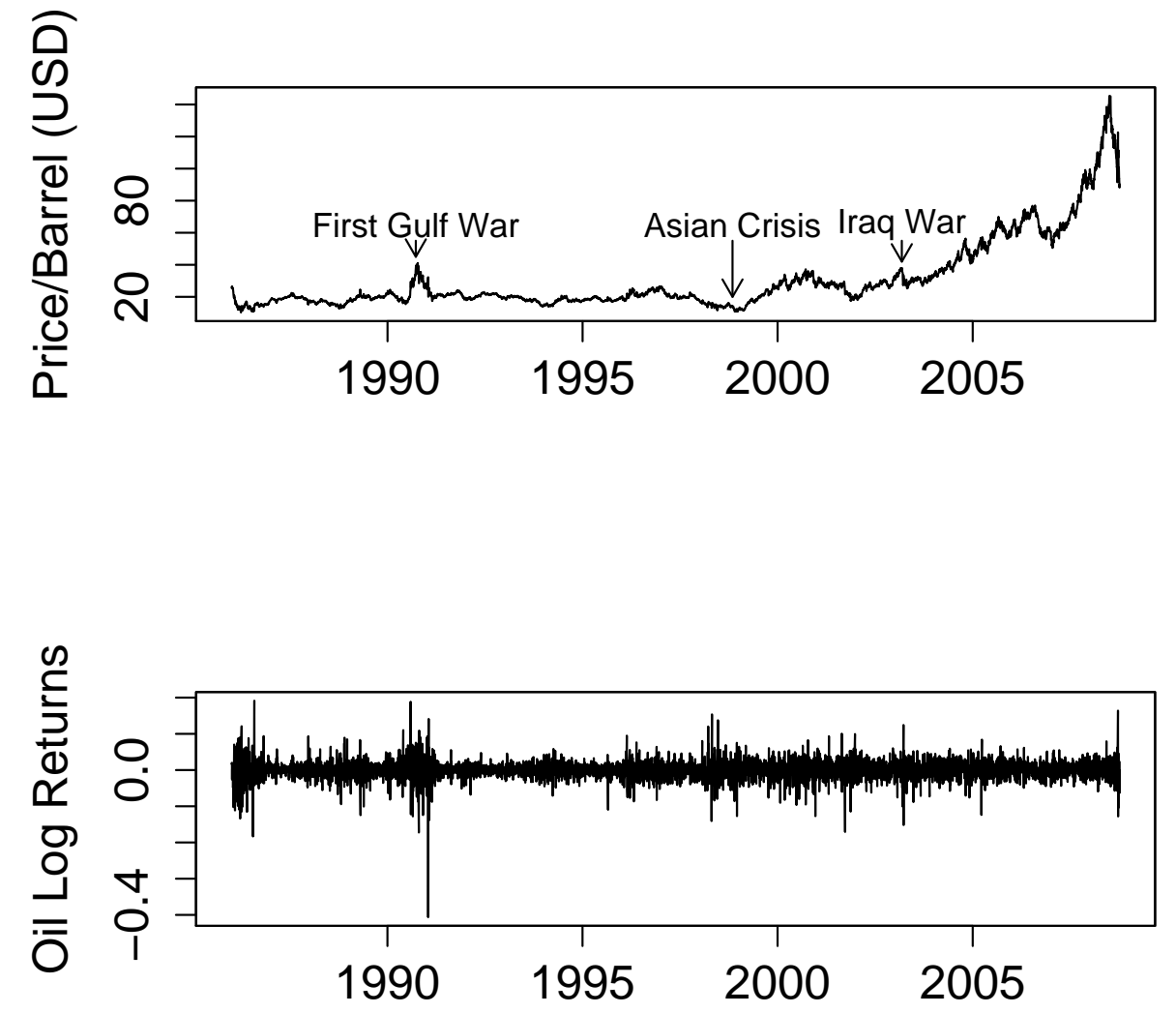

Figure 5: Top Plot: West Texas Intermediate (WTI) crude oil prices from January 2, 1986 to October 7, 2008. Bottom Plot: The daily log returns of oil prices for the same period.

We estimate next the extremal index $\theta$ of the returns using the max-spectrum, the runs $1,5,9$ and the Ferro-Segers estimators. The results are shown in Figure 6 The max-spectrum estimates of $\theta$ were obtained by setting $N_{\text {out }}=200$ and $N_{\text {in }}=1$ and using WLS. It can be seen that stable $\theta$ estimates for the right tail can be obtained at scales $j=4$ to $j=5$. Pooling these results yield a value for $\theta=0.60$ with a $95 \%$ confidence interval of $(0.55,0.65)$ based on equation $(5.24)$. It should be noted that the automatic selection procedure chooses scale $j=5$ for the right tail, which gives comparable results. The $95 \%$ confidence interval obtained from $(5.23)$ is $(0.59,0.61)$. The main reason that these confidence intervals are narrow is because they ignore the uncertainty regarding scale selection. For the left tail, we choose the median value at scales $j=5$ to $j=6$ and to obtain a pooled estimate of 0.53 with a $95 \%$ confidence interval of $(0.47,0.61)$ using (5.24) and $(0.51,0.55)$ using (5.23) and $j=5$.

A reasonably stable estimate obtained from the Ferro-Segers procedure is around 0.50 for the right tail and 0.42 for the left one. However, another choice for the left tail is 0.53 , corresponding to the range of 0.90 th to 0.92 nd quantiles. The max-spectrum and Ferro-Segers estimates are to some extent in agreement for the right tail and possibly for the left tail as well, depending on the choice of a stable range for the Ferro-Segers estimate. On the other hand, the results of the runs- 1 estimator are highly suspect. The results of the runs- 1 indicate little or no clustering of extremes (as $\hat{\theta} \approx 1$ ). The fact that runs- 1 fails to capture the clustering may be explained by the behavior of financial returns, where one extremely large positive return is commonly 

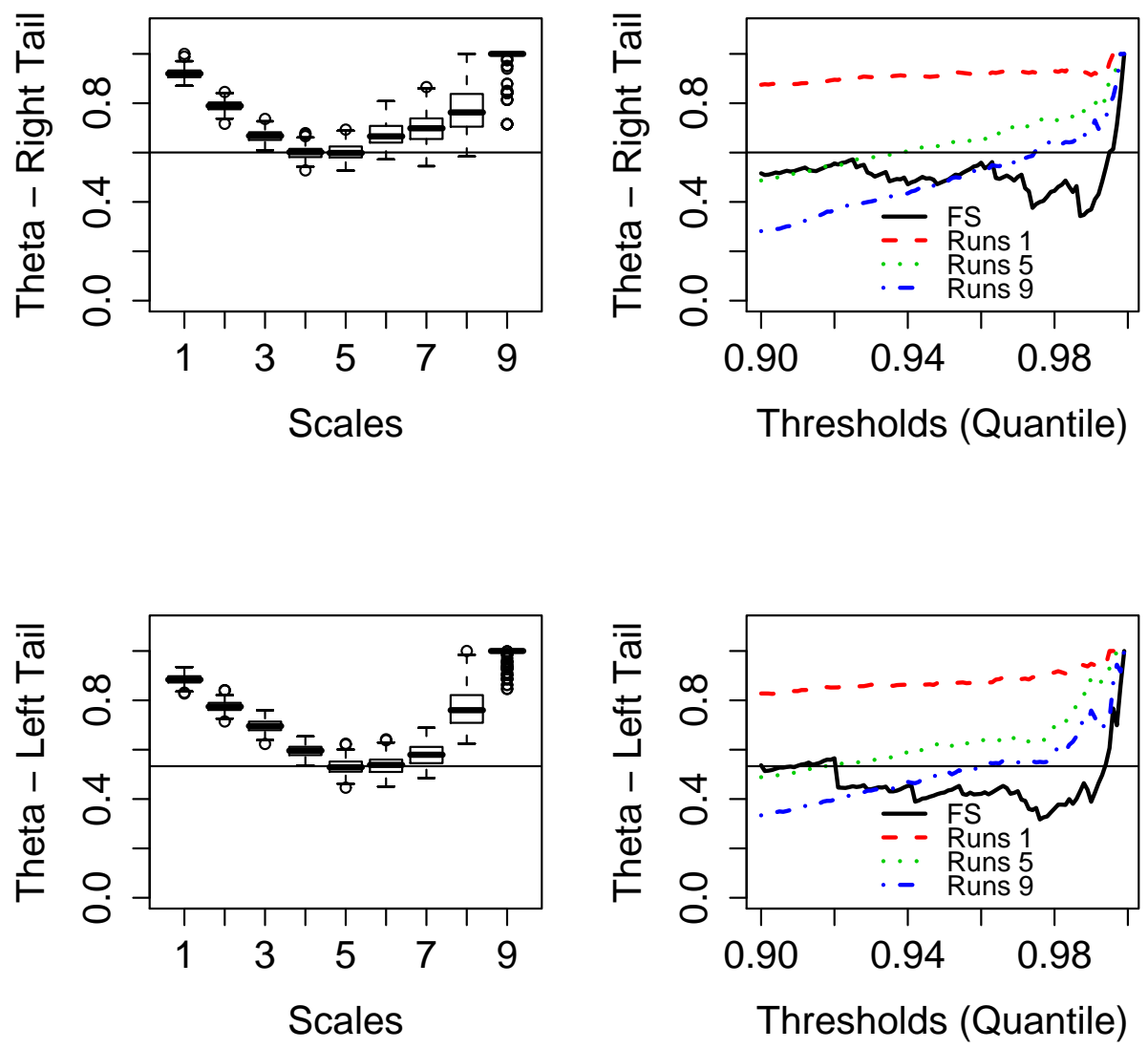

Figure 6: Top Row: Estimates of $\theta$ for the right tail. The left panel is the max spectrum estimates. The right panel is the Ferro-Segers and runs estimates. The solid horizontal line in both plots corresponds to the max spectrum point estimate of 0.60. Bottom Row: Estimates of $\theta$ for the left tail. The left panel is the max spectrum estimates. The right panel is the Ferro-Segers and runs estimates. The solid horizontal line in both plots corresponds to the max spectrum point estimate of 0.60 .

followed by a large negative return. Thus, runs- 1 often identifies clusters with a single extreme value, as in the case of independent data. Increasing the number of the run length parameter yields estimates more in agreement with the other two procedures. The results strongly suggest clustering of large losses and gains that can in turn have serious consequences in terms of risk exposure of portfolios that include WTI.

The next two examples illustrate our extremal index estimator over two financial data sets: (i) Daily returns of the S\& P 500 stock index and (ii) high-frequency, tick-by-tick volumes of a traded stock. The extremal index estimates behave differently in these two settings over the largest scales $j$. We discuss how the plot of the $\widehat{\theta}(j)$ 's, as a function of $j$, may be used to detect different regimes of clustering of extremes. For simplicity, we focus on $\widehat{\theta}(j)$ 's obtained by weighted least squares, $N_{\text {in }}=1$ and $N_{\text {out }}=200$ independent permutations of the data. The results with other choices of the parameters, or ones involving bootstrap instead of permutations are similar.

Daily S\&P 500 returns (1960-2007): Figure 7 shows the extremal index estimates of the gains and losses for the daily returns of the S\&P 500 stock index. The top panel indicates that both the gains and the losses 


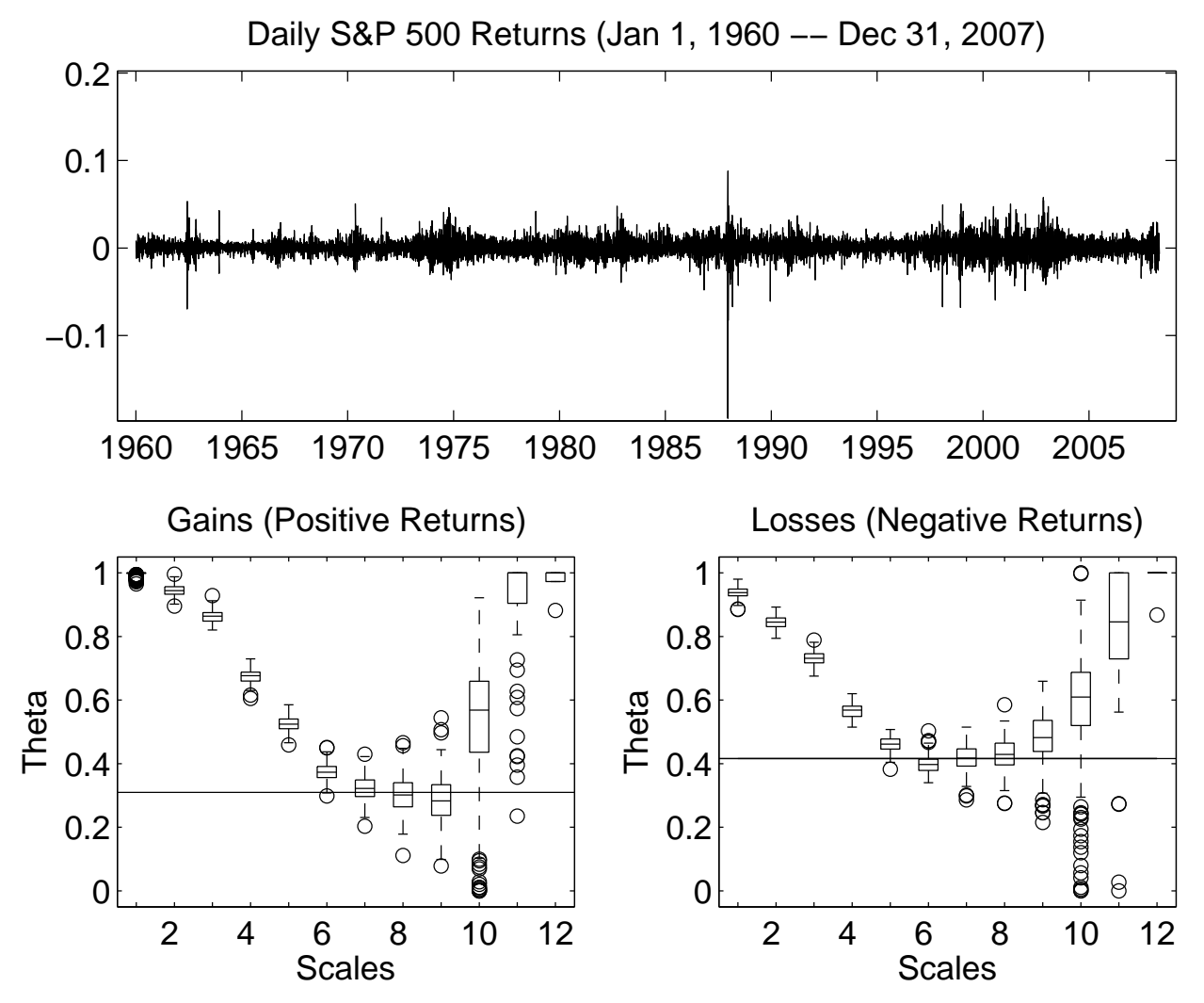

Figure 7: Top panel: S\&P 500 index (daily returns). Bottom panels: boxplots of the $\widehat{\theta}(j)$ 's obtained from $N_{\text {out }}=200$ independent permutations of the data. (The corresponding bootstrap-based versions are similar and omitted for brevity.) The left panel corresponds to the time series of positive returns (gains); the right panel to the time series of the absolute values of the negative returns (losses). Observe that the extremal index estimates over the largest scales approach 1 for both the gains and the losses.

time series have heavy tails. Indeed, max-spectrum estimates of the left- and right-tail exponents yield $\widehat{\alpha}_{\text {loss }} \approx 2.958$ and $\widehat{\alpha}_{\text {gain }} \approx 3.553$. These values confirm the common observation that the tails of the losses are slightly heavier than the tails of the gains (see e.g. Table 1 in Galbraith and Zernov (2006)). The bottom two panels on Figure 7 show boxplots of resampled estimates of the extremal index $\theta$ as a function of the scale $j$. We studied separately the time series of the gains or positive returns (left panel) and the losses (right panel).

For the gains, the box-plots stabilize at scales $j=7$ to 9 (as also confirmed by the Kruskal-Wallis analysis). As for the oil data, by pooling the $\widehat{\theta}(j)$ 's for this range of scales, we obtain $\widehat{\theta}_{\text {gains }} \approx 0.31$ with $95 \%$ confidence interval $(0.23,0.39)$ based on $(5.23)$ and scale $j=7$. The confidence interval based on (5.24) and pooling scales $j=7$ to 9 together is $(0.16,0.43)$. Similar analysis for the losses shows that the $\widehat{\theta}(j)$ 's stabilize over the range $j=6$ to 8 , and the pooled estimate is $\widehat{\theta}_{\text {loss }} \approx 0.416$. The $95 \%$ confidence interval based on (5.23) and scale $j=6$ is $(0.34,0.49)$, and the one based on the pooled scales and (5.24) is $(0.34,0.50)$. Our results are in agreement with the Ferro-Segers and runs estimates (for 200 threshold exceedences therein) of $\theta_{\text {loss }}$ reported in Figure $3 \mathrm{~b}$ of Galbraith and Zernov (2006).

Our analysis indicates that the extremal indices of both the gains and the losses time series of daily S\&P 500 returns are lower than the estimates corresponding to the Oil data set. This, as before, shows that extremes of the gains and the losses exhibit significant clustering, which can have far reaching consequences in terms of risk management. In contrast to the Oil data set, however, the left tails (losses) have slightly 

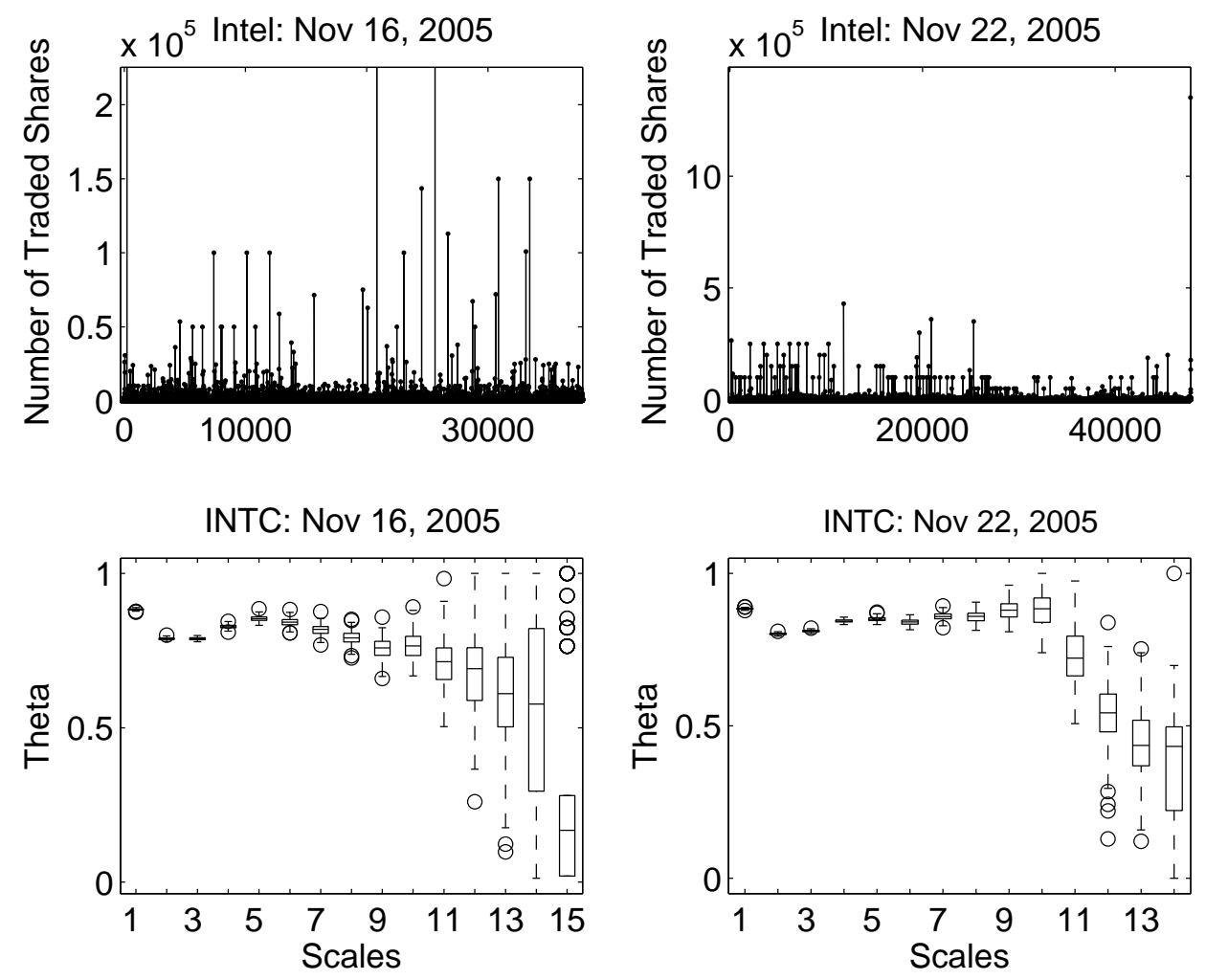

Figure 8: Top panels: High-frequency volume time series for the Intel stock during the days of Nov 16 (left panel) and Nov 22 (right panel) in 2005. The data are ordered in time and every value corresponds to the number of traded shares during one transaction. There are 75, 993 trades in Nov 16 and 119, 840 trades in Nov 22. Bottom panels: boxplots of the $\widehat{\theta}(j)$ 's obtained from $N_{\text {out }}=200$ independent permutations of the data corresponding to the top two data sets.

higher extremal index than the right tails (gains). This results in slightly more temporal clustering of the extreme gains as compared to the extreme losses. Indeed, the expected cluster sizes for the extreme gains and losses are about $1 / \widehat{\theta}_{\text {gains }} \approx 3.23$ and $1 / \widehat{\theta}_{\text {loss }} \approx 2.5$, respectively.

The above estimates yield a single value for the extremal index $\theta$ based on a judicious choice of scales. In practice, the boxplots for the entire range of available scales, however, can also give important insights. In the above analysis, we focus on the range of scales $j=6$ to 9 , which roughly corresponds to focusing on the range of probabilities $[0.9844,0.9980]$. Therefore, from a physical perspective, the extremal index estimates are useful and applicable for the extremes occurring on a time scale of up to $2^{9}=512$ trading days or up to 2 years, on the average. Over a range of 1 to 2 years, one can indeed expect that the S\&P 500 returns are approximately stationary and our theory applies. Significant structural changes and cycles in the economy, however, lead to non-stationarity over longer periods of time. Therefore, the extremal index estimates $\widehat{\theta}(j)$ 's for scales $j \geq 10$ should also be considered, but interpreted with care. Indeed, as seen from Figure 7 the estimates $\widehat{\theta}(j)$ 's approach 1 , as $j$ grows beyond 9 . For the largest scales $(j=11$ or 12$)$, the extremal indices of the gains and losses are essentially 1 . Since $\theta$ measures the degree of clustering or dependence of extremes, this suggests that the largest extremes of the S\&P 500 returns are perhaps weakly dependent or independent. Indeed, the largest extremes correspond to select few financial crashes or periods of extreme volatility. These events occur far apart in time, they do not cluster, and therefore $\widehat{\theta}(j) \approx 1$.

High-frequency Stock Volume: Figure 8 illustrates the extremal index estimators over two high-frequency 
data sets of traded volumes. The time series consist of the number of traded shares of Intel Inc. for each and every transaction occurring during two typical days of trading (Nov 16 and 22 in 2005). The data was obtained from the TAQ (trades and quotes) data base of consolidated trades from the NYSE and NASDAQ exchanges (see Wharton Research Data Service (url)). One easily sees that reasonable extremal index estimates for Nov 16 and Nov 22 are about $\widehat{\theta} \approx 0.8$. The corresponding boxplots are stable over a wide range of scales (e.g. $j=6$ to 10 ). Beyond scales $j=10$, however, one should interpret the estimates $\widehat{\theta}(j)$ 's with care. Indeed, about $2^{10}=1024$ trades of the highly liquid Intel stock occur over the time scale of about 4 to 5 minutes (depending on the time of the day and the amount of trading during the day). Over the time scale of 4 to 5 minutes, one can expect to have relatively stationary trading patterns. Longer periods of time, however, involve intra-day seasonality and other intricate non-stationarity due to arrival of new information. Therefore, the boxplots of the $\widehat{\theta}(j)$ 's involve a 'change of regime' for scales $j \geq 10$. This change of regime is relatively abrupt for the November 22 data set and gradual but systematic for the November 16 data. In both cases, the extremal index estimates drastically approach zero, as the scales become more extreme. This implies that the clustering of the largest extremes is substantially more pronounced than that of the moderate extremes. This effect is also confirmed by the top plots where extremely large volumes are traded in batches. This phenomenon is in stark contrast with the observed weakening of the clustering for the returns data in Figure 7. This difference may be attributed to the difference in the nature of the extreme traded volumes and extreme stock returns. Large returns/losses in the S\&P 500 index are global, market-wide events that are hard to control or manipulate. Extremely large volumes, on the other hand, are traded by a select individual players in the market. Typically, large transactions are split in batches and traded systematically over a longer period of time to minimize the negative effect of a large volume trade on the stock price.

\section{Concluding Remarks}

In this paper, a novel procedure for estimating the extremal index of stationary time series was introduced. It is based on scaling properties of block-maxima and on resampling. Under certain mild regularity conditions, its consistency and asymptotic normality were established for $m$-dependent time series, that provides one way of constructing confidence intervals. A more computationally intensive procedure based on resampling is also presented for the same task. A comprehensive simulation study shows the competitive nature of the proposed estimator. Finally, the estimator is illustrated on a number of financial data sets that also demonstrate additional diagnostic features of various statistical plots based on the new estimator.

\section{References}

Beirlant, J., Goegebeur, Y., Segers, J., and Teugels, J. (2004). Statistics of Extremes. Wiley.

Embrechts, P., Kluppelberg, C., and Mikosch, T. (1997). Modelling Extremal Events. Springer.

Ferro, C. A. T. and Segers, J. (2003). Inference for clusters of extreme values. J. R. Stat. Soc. Ser. B Stat. Methodol., $\mathbf{6 5}(2), 545-556$.

Finkenstädt, B. and Rootzén, H., editors (2004). Extreme Values in Finance, Telecommunications, and the Environment, Volume 99 of Monographs on Statistics and Applied Probability. Chapman and Hall/CRC.

Galbraith, J. W. and Zernov, S. (2006). Extreme dependence in the NASDAQ and S\&P 500 composite indexes. Preprint. http://www.mcgill.ca/files/economics/extremedependencein.pdf.

Geman, H. (2005). Commodities and Commodity Derivatives : Modelling and Pricing for Agriculturals, Metals and Energy. Wiley. 
Hamidieh, K., Stoev, S., and Michailidis, G. (2007). On the estimation of the extremal index based on scaling and resampling. Technical Report 462, Department of Statistics, The University of Michigan.

Hsing, T. (1993). Extremal index estimation for a weakly dependent stationary sequence. The Annals of Statistics, 21, 2043-2071.

Hsing, T., Husler, J., and Leadbetter, M. (1988). On the exceedance point process for a stationary sequence. Probability Theory and Related Fields, 78, 97-112.

Johnson, N.L., Kotz, S. and Balakrishnan, N. (1994). Continuous Univariate Distributions, Wiley Series in Probability and Mathematical Statistics, Volume 2.

Laurini, F. (2004). Clusters of extreme observations and extremal index estimate in GARCH processes. Studies in Nonlinear Dynamics and Econometrics, 8(4).

Leadbetter, M. R., Lindgren, G., and Rootzén, H. (1983). Extremes and Related Properties of Random Sequences and Processes. Springer.

Longin, F. (2000). From value at risk to stress testing: the extreme value approach. Journal of Banking and Finance, 24, 1097-1130.

O'Brien, G. (1987). Extreme values for stationary and Markov sequences. The Annals of Probability, 15, 281-291.

Smith, R. L. and Weissman, I. (1994). Estimating the extremal index. Journal of the Royal Statistical Society, Series $B, \mathbf{5 6}, 515-528$.

Stoev, S. A., Michailidis, G., and Taqqu, M. S. (2006). Estimating heavy-tailed exponents through max self-similarity. Technical Report 445, The University of Michigan.

Weissman, I. and Novak, S. Y. (1998). On blocks and runs estimators of the extremal index. J. Statist. Plann. Inference, 66(2), 281-288.

Wharton Research Data Service (url) (2007). Wharton School of Management, Universty of Pennsylvania. https://wrds.wharton. upenn.edu/.

\section{Appendix}

Proposition 7.1 Suppose that $f:(0, \infty) \rightarrow \mathbb{R}$ is an absolutely continuous function on any compact interval $[a, b] \subset$ $(0, \infty)$, and such that $f(x)=f\left(x_{0}\right)+\int_{x_{0}}^{x} f^{\prime}(u) d u, x>0$ for some (any) $x_{0}>0$.

Let for some $m \in \mathbb{R}$ and $\delta>0$,

$$
\begin{gathered}
x^{m}|f(x)|+\operatorname{esssup}_{0<y \leq x} y^{m}\left|f^{\prime}(y)\right| \longrightarrow 0, \quad \text { as } x \downarrow 0, \\
x^{-\alpha}|f(x)|+x^{1+\delta} \operatorname{essup}_{y \geq x} y^{-\alpha}\left|f^{\prime}(y)\right| \longrightarrow 0, \quad \text { as } x \rightarrow \infty .
\end{gathered}
$$

Suppose also that the time series $X=\left\{X_{n}\right\}_{n \in \mathbb{Z}}$ satisfies Conditions 1 and 2 , where $c_{1}(x)$ is such that:

$$
\int_{1}^{\infty} c_{1}(x) x^{-\alpha}\left|f^{\prime}(x)\right| d x<\infty .
$$

Then, $E\left|f\left(M_{n}\right)\right|<\infty$, for all sufficiently large $n \in \mathbb{N}$, and for some $C_{f}>0$, independent of $n$,

$$
\left|\mathbb{E} f\left(M_{n} / n^{1 / \alpha}\right)-\mathbb{E} f(Z)\right| \leq C_{f} n^{-\beta},
$$

where $Z$ is an $\alpha$-Fréchet variable with scale coefficient $\sigma:=c_{X}^{1 / \alpha}$. 
Proof: The proof is similar to the proof of Theorem 3.1 in Stoev et al. (2006). Indeed, as in the above reference, one can show that $\mathbb{E}|f(Z)|<\infty$ and $\mathbb{E}\left|f\left(M_{n}\right)\right|<\infty$, for all sufficiently large $n$. Further, by using the conditions (7.25) and (7.26) and integration by parts, we have that

$$
\mathbb{E} f\left(M_{n} / n^{1 / \alpha}\right)-\mathbb{E} f(Z)=\int_{0}^{\infty}\left(G(x)-F_{n}(x)\right) f^{\prime}(x) d x,
$$

where $F_{n}(x):=\mathbb{P}\left\{M_{n} / n^{1 / \alpha} \leq x\right\}$ and $G(x)=\mathbb{P}\{Z \leq x\}$. Since $F_{n}(x)=e^{-c(n, x) x^{-\alpha}}$, by the mean value theorem, we have

$$
\begin{aligned}
\left|G(x)-F_{n}(x)\right| & =\left|e^{-c_{X} x^{-\alpha}}-e^{-c(n, x) x^{-\alpha}}\right| \leq\left|c(n, x)-c_{X}\right| x^{-\alpha} e^{-\min \left\{\theta c_{X}, c(n, x)\right\} x^{-\alpha}} \\
& \leq n^{-\beta} c_{1}(x) x^{-\alpha}\left(e^{-c_{2} x^{-(\alpha-\gamma)}}+e^{-\theta c_{X} x^{-\alpha}}\right),
\end{aligned}
$$

where in the last inequality, we used Relations (3.12) and (3.13).

Thus, by (7.29), we have that

$$
\begin{aligned}
\left|\mathbb{E} f\left(M_{n} / n^{1 / \alpha}\right)-\mathbb{E} f(Z)\right| & \leq n^{-\beta} \int_{0}^{\infty} c_{1}(x) x^{-\alpha}\left|f^{\prime}(x)\right|\left(e^{-c_{2} x^{-(\alpha-\gamma)}}+e^{-c_{X} x^{-\alpha}}\right) d x \\
& =: n^{-\beta}\left(\int_{0}^{1}+\int_{1}^{\infty}\right) .
\end{aligned}
$$

The last integral is finite. Indeed, since the exponential terms above are bounded, Relation (7.27) implies that the integral " $\int_{1}^{\infty}$ " is finite. On the other hand, conditions (3.12) and (7.25) imply that, $c_{1}(x)\left|f^{\prime}(x)\right|=\mathcal{O}\left(x^{-R}\right), x \downarrow 0$, for some $R \in \mathbb{R}$. However, for all $p>0$, we have $\left(e^{-c_{2} x^{-(\alpha-\gamma)}}+e^{-c_{X} x^{-\alpha}}\right)=o\left(x^{p}\right), x \downarrow 0$, since $\alpha-\gamma>0$. This implies that the integral in " $\int_{0}^{1}$ " in (7.30) is also finite. This completes the proof of (7.28).

Proposition 7.2 Let $X=\left\{X_{k}\right\}_{k \in \mathbb{Z}}$ be a strictly stationary time series which satisfies Conditions 1 and 2 in Section 3 above. Suppose that $\int_{1}^{\infty} c_{1}(x) x^{-\alpha-1+\delta} d x<0$, for some $\delta>0$.

Then, with $M_{n}:=\max _{1 \leq k \leq n} X_{k}$, we have $\mathbb{E}\left|\ln \left(M_{n}\right)\right|^{p}<\infty$, for all $p>0$ and all sufficiently large $n \in \mathbb{N}$. Moreover, for any $p>0$ and $k \in \mathbb{N}$, we have:

$$
\left.|\mathbb{E}| \ln \left(M_{n} / n^{1 / \alpha}\right)\right|^{p}-\mathbb{E}|\ln (Z)|^{p} \mid=\mathcal{O}\left(n^{-\beta}\right), \quad \text { and } \quad\left|\mathbb{E}\left(\ln \left(M_{n} / n^{1 / \alpha}\right)\right)^{k}-\mathbb{E}(\ln (Z))^{k}\right|=\mathcal{O}\left(n^{-\beta}\right),
$$

as $n \rightarrow \infty$, where $Z$ is an $\alpha$-Fréchet random variable with scale coefficient $\theta^{1 / \alpha} c_{X}^{1 / \alpha}$.

Proof: It is enough to show that the functions $f(x):=|\ln (x)|^{p}$ and $f(x):=(\ln (x))^{k}, p>0, k \in \mathbb{N}$ satisfy the conditions of Proposition 7.1. In the first case, for example, $\left|f^{\prime}(x)\right|=p x^{-1}|\ln (x)|^{p-1}, x>0$. Therefore, the assumption $\int_{1}^{\infty} c_{1}(x) x^{-\alpha-1+\delta} d x<\infty$ implies (7.27), since $|\ln (x)|^{p-1} \leq \operatorname{const} x^{\delta}$, for all $x \in[1, \infty)$. The conditions (7.25) and (7.26) are also fulfilled in this case, and hence Proposition 7.1 yields the desired order of convergence. The functions $f(x)=(\ln (x))^{k}, k \in \mathbb{N}$ can be treated similarly.

Note that, under the assumptions of Proposition 7.2, we readily obtain:

$$
\mathbb{E}\left(Y_{j}-j / \alpha\right) \equiv \mathbb{E} \log _{2}\left(D(j, k) / 2^{j / \alpha}\right)=\mathbb{E} \log _{2}\left(\theta^{1 / \alpha} c_{X}^{1 / \alpha} Z_{1}\right)+\mathcal{O}\left(1 / 2^{j \beta}\right),
$$

as $j \rightarrow \infty$, where $Z_{1}$ is a standard $\alpha$-Fréchet variable. This important fact is used in the proofs of the asymptotic results given below.

Proof of Proposition 3.1; Recall that by [2.2,

$$
D(j, k):=\bigvee_{i=1}^{2^{j}} X_{2^{j}(k-1)+i} \quad \text { and introduce } \quad \widetilde{D}(j, k):=\bigvee_{i=1}^{2^{j}-m} X_{2^{j}(k-1)+i}
$$

Observe that $\widetilde{D}(j, k), k=1, \ldots, n_{j}\left(n_{j}=\left\lfloor n / 2^{j}\right\rfloor\right)$ are independent in $k$ since they are "separated by $m$ " blockmaxima of the $m$-dependent process $X$. 
Recall also that by 2.4

$$
Y_{j}:=\frac{1}{n_{j}} \sum_{k=1}^{n_{j}} \log _{2} D(j, k) \quad \text { and introduce the statistics } \quad \widetilde{Y}_{j}:=\frac{1}{n_{j}} \sum_{k=1}^{n_{j}} \log _{2} \widetilde{D}(j, k) .
$$

We first establish Relation 3.17). Let

$$
\hat{H}=\sum_{i=0}^{\ell} w_{i} Y_{i+j(n)}, \quad \text { and } \quad \widetilde{H}=\sum_{i=0}^{\ell} w_{i} \widetilde{Y}_{i+j(n)},
$$

so that $\hat{\alpha}(j)$ in (3.15) equals $1 / \hat{H}$. The weights $w_{i}$ 's, the range $\ell$ and the quantity $j(n)$ are described in Section 3 ,

To prove that $\hat{\alpha}(j)-\alpha=\mathcal{O}_{P}\left(a_{n}\right), n \rightarrow \infty$, for some $a_{n} \rightarrow 0$, it suffices to show that $\mathbb{E}(\hat{H}-H)^{2}=\mathcal{O}\left(a_{n}^{2}\right)$, where $H:=1 / \alpha$. Observe that by adding and subtracting the term $\widetilde{H}$, and by applying the inequality $(a+b)^{2} \leq 2 a^{2}+2 b^{2}$, we get

$$
\begin{aligned}
\mathbb{E}(\hat{H}-H)^{2} \leq 2 \mathbb{E}(\hat{H}-\widetilde{H})^{2}+2 \mathbb{E}(\widetilde{H}-H)^{2}= & 2 \operatorname{Var}(\hat{H}-\widetilde{H})+2(\mathbb{E} \hat{H}-\mathbb{E} \widetilde{H})^{2}+2 \mathbb{E}(\widetilde{H}-H)^{2} \\
=: & 2 A_{1}+2 A_{2}+2 A_{3},
\end{aligned}
$$

where in the last relation we also used the fact that $\mathbb{E} \xi^{2}=\operatorname{Var}(\xi)+(\mathbb{E} \xi)^{2}$.

We will first show that $A_{1}=o\left(1 / n_{j}\right)$ in (7.34) is negligible. Indeed, by (7.33), we have

$$
\hat{H}-\widetilde{H}=\sum_{i=0}^{\ell} w_{i}\left(Y_{i+j(n)}-\widetilde{Y}_{i+j(n)}\right)
$$

and thus by using the inequality $\operatorname{Var}\left(\xi_{0}+\cdots+\xi_{\ell}\right) \leq(\ell+1)^{2}\left(\operatorname{Var}\left(\xi_{0}\right)+\cdots+\operatorname{Var}\left(\xi_{\ell}\right)\right)$, we get $\operatorname{Var}(\hat{H}-\widetilde{H}) \leq$ $(1+\ell)^{2} \sum_{i=0}^{\ell} w_{i}^{2} \operatorname{Var}\left(Y_{i+j(n)}-\widetilde{Y}_{i+j(n)}\right)$. Thus, by Lemma7.1 below, since $\ell$ is fixed,

$$
\operatorname{Var}(\hat{H}-\widetilde{H}) \leq \frac{\text { const }}{n_{j}} \sum_{i=0}^{\ell} \operatorname{Var}\left(\log _{2} D(i+j(n), 1)-\log _{2} \widetilde{D}(i+j(n), 1)\right),
$$

where $n_{j}=n / 2^{j(n)}$. Lemmas 7.2 and 7.3 on the other hand, yield

$$
\operatorname{Var}(\hat{H}-\widetilde{H})=o\left(1 / n_{j}\right), \quad \text { as } n \rightarrow \infty .
$$

Now, we focus on the term $A_{2}$ in (7.34). By 7.35, we have

$$
\begin{aligned}
\sqrt{A_{2}}= & \sum_{i=0}^{\ell} w_{i}\left(\mathbb{E} Y_{i+j(n)}-\mathbb{E} \widetilde{Y}_{i+j(n)}\right)=\mathbb{E} \sum_{i=0}^{\ell} w_{i} \log _{2}\left(D(i+j(n), 1) / 2^{(i+j(n)) / \alpha}\right) \\
& -\mathbb{E} \sum_{i=0}^{\ell} w_{i} \log _{2}\left(\widetilde{D}(i+j(n), 1) / 2^{(i+j(n)) / \alpha}\right) \\
= & \sum_{i=0}^{\ell} w_{i} \mathbb{E} \log _{2}(Z)+o\left(1 / 2^{j(n) \beta}\right) \\
& -\sum_{i=0}^{\ell} w_{i}\left(\mathbb{E} \log _{2}\left(\widetilde{D}(i+j(n), 1) /\left(2^{i+j(n)}-m\right)^{1 / \alpha}\right)-\frac{1}{\alpha} \log _{2}\left(\left(2^{i+j(n)}-m\right) / 2^{i+j(n)}\right)\right),
\end{aligned}
$$

where the last relation follows from 7.31 and where $Z$ is an $\alpha$-Fréchet variable with scale coefficient $\left(\theta c_{X}\right)^{1 / \alpha}$. Now, since $\widetilde{D}(i+j(n), 1) /\left(2^{i+j(n)}-m\right)^{1 / \alpha}$ is a properly normalized block-maximum (recall $(7.32$ ) above), by Relation (7.31), we further have that

$$
\begin{aligned}
\sqrt{A_{2}} & =\sum_{i=0}^{\ell} w_{i} \mathbb{E} \log _{2}(Z)-\sum_{i=0}^{\ell} w_{i} \mathbb{E} \log _{2}(Z)+o\left(1 / 2^{j(n) \beta}\right)+\mathcal{O}\left(\log _{2}\left(1-m / 2^{j(n)}\right)\right. \\
& =o\left(1 / 2^{j(n) \beta}\right)+\mathcal{O}\left(1 / 2^{j(n)}\right)
\end{aligned}
$$


as $j(n) \rightarrow \infty$, since $\log _{2}(1-x)=\mathcal{O}(x), x \rightarrow 0$. We thus have,

$$
A_{2}=\mathcal{O}\left(1 / 2^{j(n) \min \{1, \beta\}}\right), \quad \text { as } j(n) \rightarrow \infty .
$$

Consider now the term $A_{3}$ in (7.34). As above, we have

$$
\mathbb{E}(\widetilde{H}-H)^{2}=\operatorname{Var}(\widetilde{H}-H)+(\mathbb{E} \widetilde{H}-H)^{2}=: A_{3}^{\prime}+A_{3}^{\prime \prime},
$$

and as in (7.36), we get $A_{3}^{\prime} \leq(\ell+1)^{2} \sum_{i=0}^{\ell} w_{i} \operatorname{Var}\left(\widetilde{Y}_{i+j(n)}\right)=o\left(1 / n_{j}\right)=o\left(2^{j(n)} / n\right)$, as $n_{j} \rightarrow \infty$. Also, as argued above, since $\sum_{i=0}^{\ell} w_{i}(i+j(n)) / \alpha=1 / \alpha \equiv H$, we obtain

$$
\mathbb{E} \widetilde{H}-H=\sum_{i=0}^{\ell} w_{i}\left(\mathbb{E} \log _{2} \widetilde{D}(i+j(n), 1)-(i+j(n)) / \alpha\right)=\mathcal{O}\left(1 / 2^{j(n) \min \{1, \beta\}}\right),
$$

as $j(n) \rightarrow \infty$ (see (7.38) above). By combining the bounds for terms $A_{1}, A_{2}$ and $A_{3}$ in (7.37), (7.38) and the last two relations, we obtain

$$
\hat{H}=H+\mathcal{O}_{P}\left(1 / 2^{j(n) \min \{1, \beta\}}\right)+\mathcal{O}_{P}\left(2^{j(n) / 2} / n^{1 / 2}\right), \quad \text { as } j(n), n / 2^{j(n)} \rightarrow \infty .
$$

This completes the proof of the first asymptotic relation in 3.17).

The proof of the second asymptotic relation in (3.17) is simpler. By introducing the quantity $\widetilde{C}(j):=\widetilde{Y}_{j}-j / \alpha$, we have

$$
C(j)-\widetilde{C}(j)=Y_{j}-\widetilde{Y}_{j}=\frac{1}{n_{j}} \sum_{k=1}^{n_{j}} \log _{2}(D(j, k) / \widetilde{D}(j, k)) .
$$

One can similarly show that $\operatorname{Var}(C(j)-\widetilde{C}(j))$ is of order $o\left(1 / n_{j}\right)$, as $n \rightarrow \infty$. Thus, the order of $C(j)-C$ is dictated by the orders of the bias and standard error for the quantity $\widetilde{C}(j)$. These can be handled as the terms $A_{2}$ and $A_{3}$ in (7.34).

The following three lemmas were used in the proof Proposition 3.1

Lemma 7.1 Under the conditions of Proposition 3.1 for all $j>\log _{2} m$, we have

$$
\operatorname{Var}\left(Y_{j}-\widetilde{Y}_{j}\right) \leq \frac{3}{n_{j}} \operatorname{Var}\left(\log _{2}(D(j, 1) / \widetilde{D}(j, 1))\right) .
$$

Proof: For notational simplicity, let $\xi_{k}:=\log _{2}(D(j, k) / \widetilde{D}(j, k)), k=1, \ldots, n_{j}$. We have, by the stationarity of $\xi_{k}$ in $k$, that

$$
\operatorname{Var}\left(Y_{j}-\widetilde{Y_{j}}\right)=\frac{1}{n_{j}} \operatorname{Var}\left(\xi_{1}\right)+\frac{2}{n_{j}^{2}} \sum_{k=1}^{n_{j}-1}\left(n_{j}-k\right) \operatorname{Cov}\left(\xi_{k+1}, \xi_{1}\right) .
$$

Note that $\xi_{k+1}=\log _{2}(D(j, 1+k) / \widetilde{D}(j, 1+k))$ and $\xi_{1}=\log _{2}(D(j, 1) / \widetilde{D}(j, 1))$ are independent if $k>1$. Indeed, this follows from the fact that the process $X$ is $m$-dependent, and since $\xi_{k+1}$ and $\xi_{1}$ depend on blocks of the data separated by at least $2^{j}>m$ lags. Therefore, only the lag-1 covariances in the above sum will be non-zero and hence

$$
\operatorname{Var}\left(Y_{j}-\widetilde{Y_{j}}\right) \leq \frac{1}{n_{j}} \operatorname{Var}\left(\xi_{1}\right)+\frac{2}{n_{j}}\left|\operatorname{Cov}\left(\xi_{2}, \xi_{1}\right)\right| \leq \frac{3}{n_{j}} \operatorname{Var}\left(\xi_{1}\right),
$$

since by the Cauchy-Schwartz inequality we have $\left|\operatorname{Cov}\left(\xi_{2}, \xi_{1}\right)\right| \leq \operatorname{Var}\left(\xi_{2}\right)^{1 / 2} \operatorname{Var}\left(\xi_{1}\right)^{1 / 2}=\operatorname{Var}\left(\xi_{1}\right)$. This completes the proof of the lemma.

Lemma 7.2 For $D(j, k)$ and $\widetilde{D}(j, k)$, defined in (7.32) above, for any fixed $k$, we have $D(j, k) / \widetilde{D}(j, k) \stackrel{P}{\longrightarrow} 1$, as $j \rightarrow \infty$. 
Proof: Let $\delta \in(0,1 / \alpha)$ be arbitrary and observe that

$$
\mathbb{P}\{D(j, k) / \widetilde{D}(j, k)<1\}=\mathbb{P}\{R>\widetilde{D}(j, k)\} \leq \mathbb{P}\left\{R>2^{j \delta}\right\}+\mathbb{P}\left\{2^{j \delta}>\widetilde{D}(j, k)\right\},
$$

where $R=\max _{1 \leq i \leq m} X_{2^{j}(k-i)+1}$. Now, by stationarity,

$$
\mathbb{P}\left\{R>2^{j \delta}\right\}=\mathbb{P}\left\{\max _{1 \leq i \leq m} X_{i}>2^{j \delta}\right\} \rightarrow 0, \text { as } j \rightarrow \infty .
$$

On the other hand, Relation (3.12) implies that $2^{-j / \alpha} \widetilde{D}(j, k) \stackrel{d}{\rightarrow} Z$, as $n \rightarrow \infty$, where $Z$ is a non-degenerate $\alpha-$ Fréchet variable. Thus, since $\delta \in(0,1 / \alpha)$, we have that

$$
\mathbb{P}\left\{2^{j \delta}>\widetilde{D}(j, k)\right\} \rightarrow 0, \quad \text { as } j \rightarrow \infty .
$$

The last two convergences and the inequality 7.39 imply that $\mathbb{P}\{D(j, k) / \widetilde{D}(j, k)<1\} \rightarrow 0, j \rightarrow \infty$. Since trivially $\mathbb{P}\{D(j, k) / \widetilde{D}(j, k)>1\}=1$, we obtain $D(j, k) / \widetilde{D}(j, k)$ converges in distribution to the constant 1 , as $j \rightarrow \infty$. This completes the proof since convergence in distribution to a constant implies convergence in probability.

Lemma 7.3 The set of random variables $\left|\log _{2}(D(j, k) / \widetilde{D}(j, k))\right|^{p}, j, k \in \mathbb{N}$ is uniformly integrable, for all $p>0$, where $D(j, k)$ and $\widetilde{D}(j, k)$ are defined in $(7.32)$.

Proof: Let $q>p$ be arbitrary. By using the inequality $|x+y|^{q} \leq 2^{q}\left(|x|^{q}+|y|^{q}\right), \quad x, y \in \mathbb{R}$, we get

$$
\mathbb{E}\left|\log _{2} \frac{D(j, k)}{\widetilde{D}(j, k)}\right|^{q} \leq 2^{q} \mathbb{E}\left|\log _{2}\left(D(j, k) / 2^{j / \alpha}\right)\right|^{q}+2^{q} \mathbb{E}\left|\log _{2}\left(\widetilde{D}(j, k) / 2^{j / \alpha}\right)\right|^{q} .
$$

In view of Proposition 7.2 applied to the block-maxima $D(j, k)$ and $\widetilde{D}(j, k)$, we obtain

$$
\mathbb{E}\left|\log _{2}\left(D(j, k) / 2^{j / \alpha}\right)\right|^{q}=\mathbb{E}\left|\log _{2}\left(M_{2^{j}} / 2^{j / \alpha}\right)\right|^{q} \longrightarrow \text { const, } \text { as } j \rightarrow \infty .
$$

Thus the set $\left\{\mathbb{E}\left|\log _{2}\left(D(j, k) / 2^{j / \alpha}\right)\right|^{q}, j, k \in \mathbb{N}\right\}$ is bounded. We similarly have that the set $\left\{\mathbb{E}\left|\log _{2}\left(\widetilde{D}(j, k) / 2^{j / \alpha}\right)\right|^{q}\right\}_{j, k \in \mathbb{N}}$ is bounded since $\log _{2}\left(2^{j}-m\right) \sim j, j \rightarrow \infty$, for any fixed $m$.

We have thus shown that

$$
\sup _{j, k \in \mathbb{N}} \mathbb{E}\left|\log _{2} \frac{D(j, k)}{\widetilde{D}(j, k)}\right|^{q}<\infty
$$

for $q>p$, which yields the desired uniform integrability.

Proof of Lemma 3.1; Suppose that the indices $i_{1}, \ldots, i_{k}$ are drawn without replacement. Let $A_{1}=\Omega$ and

$$
A_{j}:=\left\{\omega \in \Omega:\left|i_{j^{\prime}}(\omega)-i_{j^{\prime \prime}}(\omega)\right| \geq m, \text { for all } j^{\prime} \neq j^{\prime \prime}, 1 \leq j^{\prime}, j^{\prime \prime} \leq j\right\},
$$

for $j \geq 2$, that is, $A_{j}$ is the event that the first $j$ random indices are spaced further away from each other by at least $m$ lags. By convention, we let $A_{1}$ denote the almost sertain event, so that $\mathbb{P}\left(A_{1}\right)=1$.

We need to show $\mathbb{P}\left(A_{k}\right) \geq 1-m k^{2} /(n-k)$. Note that, since $\mathbb{P}\left(A_{1}\right)=1$ by convention, for all $j \geq 1$, we obtain

$$
\mathbb{P}\left(A_{j+1}\right)=\mathbb{P}\left(A_{j+1} \mid A_{j}\right) \mathbb{P}\left(A_{j}\right) \geq(1-2 m j /(n-j)) \mathbb{P}\left(A_{j}\right) .
$$

Indeed, the probability $\mathbb{P}\left(A_{j+1} \mid A_{j}\right)$ of choosing the index $i_{j+1}$ to be within $m$ lags from at least one of the chosen $j$ indices $i_{1}, \ldots, i_{j}$ is at most $2 m j /(n-j)$. Thus,

$$
\mathbb{P}\left(A_{k}\right)=\prod_{j=1}^{k-1} \mathbb{P}\left(A_{j+1} \mid A_{j}\right) \mathbb{P}\left(A_{1}\right) \geq \prod_{j=1}^{k-1}(1-2 m j /(n-j)) .
$$

Now, by the inequality $\prod_{j=1}^{k-1}\left(1-x_{j}\right) \geq 1-\sum_{j=1}^{k-1} x_{j}$, valid for all $x_{j} \in[0,1]$, we obtain

$$
\mathbb{P}\left(A_{k}\right) \geq 1-\sum_{j=1}^{k-1} 2 m j /(n-j) \geq 1-m k(k-1) /(n-k)>1-m k^{2} /(n-k) .
$$


The case when the indices are drawn with replacement is similar.

Proof of Theorem 3.1; Consider either a bootstrap or a permutation sample $X_{l}^{*}=X_{i_{l}}, l=1, \ldots, k$, where $i_{1}, \ldots, i_{k}$ are randomly chosen indices from $\{1, \ldots, n\}$, independently from the original data $X_{1}, \ldots, X_{n}$. In the case of bootstrap these indices are chosen with replacement and in the case of permutations - without replacement, respectively.

Let the event $A_{k}$ be defined as in (7.40), which corresponds to the indices being spaced by at least $m$-lags away from each other. Thus, since the time series $X=\left\{X_{i}\right\}_{i \in \mathbb{Z}}$ is $m$-dependent,

$$
\left(X_{1}^{*}, \cdots, X_{k}^{*}\right) 1_{A_{k}} \stackrel{D}{=}\left(\widetilde{X}_{1}, \cdots, \widetilde{X}_{k}\right) 1_{A_{k}},
$$

where $\widetilde{X}_{l}, l=1, \ldots, k$ are iid random variables with the same distribution as the $X_{n}$ 's which are independent from the event $A_{k}$. Observe that the event $A_{k}$ is also independent from the time series $X$ since it depends only on the random indices $i_{1}, \ldots, i_{k}$. Further, note that in the last relation, we have only equality in distribution and not equality almost surely.

Now, by Lemma 3.1, we have $\mathbb{P}\left(A_{k}\right) \rightarrow 1$, as $k \rightarrow \infty$, since $k(n)=o(\sqrt{n})$. Thus, Lemma 7.4 implies that any statistic based on the bootstrap or the randomly permuted sample will have the same limiting distribution as the corresponding statistic based on the iid sample $\left\{\widetilde{X}_{l}\right\}_{1 \leq l \leq k}$.

Let $\widetilde{C}^{*}(j)=\widetilde{Y}_{j}-j / \alpha$ be defined as the quantity $C^{*}(j)$ in $(3.16)$, but where now $\widetilde{Y}_{j}$ is the max-spectrum based on the iid data $\widetilde{X}_{1}, \ldots, \widetilde{X}_{k}$. Theorem 4.1 in Stoev et al. (2006) implies that

$$
\sqrt{k_{j}}\left(\widetilde{C}^{*}(j)-C^{*}\right) \stackrel{D}{\longrightarrow} \mathcal{N}\left(0, \sigma_{C^{*}}^{2}\right), \quad \text { as } k \rightarrow \infty,
$$

where $\sigma_{C^{*}}^{2}$ is as in Theorem 3.1 As argued above, Lemma 7.4 and Relation (7.43) imply (3.18), which completes the proof of the theorem.

Lemma 7.4 Let $X_{n}, X$ and $Y_{n}$ be real random variables such that $X_{n} \stackrel{D}{\rightarrow} X$, as $n \rightarrow \infty$. Let also $A_{n}$ and $B_{n}$ be some events such that $Y_{n} 1_{B_{n}} \stackrel{D}{=} X_{n} 1_{A_{n}}$. If $\mathbb{P}\left(A_{n}\right)=\mathbb{P}\left(B_{n}\right) \rightarrow 1, n \rightarrow \infty$, then $Y_{n} \stackrel{D}{\rightarrow} X$, as $n \rightarrow \infty$.

Proof: Let $f: \mathbb{R} \rightarrow \mathbb{R}$ be an arbitrary bounded and continuous function. Since $\mathbb{E}\left|f\left(Y_{n}\right) 1_{B_{n}^{c}}\right| \leq \operatorname{const} \mathbb{P}\left(B_{n}^{c}\right)=o(1)$, as $n \rightarrow \infty$, we have

$$
\mathbb{E} f\left(Y_{n}\right)=\mathbb{E} f\left(Y_{n}\right) 1_{B_{n}}+o(1)=\mathbb{E} f\left(X_{n}\right) 1_{A_{n}}+o(1)=\mathbb{E} f\left(X_{n}\right)+o(1), \quad \text { as } n \rightarrow \infty .
$$

This shows that $\lim _{n \rightarrow \infty} \mathbb{E} f\left(Y_{n}\right)=\lim _{n \rightarrow \infty} \mathbb{E} f\left(X_{n}\right)$, which completes the proof.

Proof of Theorem 3.2, Recall relation (3.16) and observe that by Proposition 3.1, we have

$$
\hat{\alpha}(j)=\alpha+\mathcal{O}_{P}\left(b_{n}\right), \quad \text { and } \quad C(j)=C+\mathcal{O}_{P}\left(b_{n}\right),
$$

as $n \rightarrow \infty$, where $b_{n}=1 / 2^{j(k(n)) \min \{1, \beta\}}+2^{j(k(n)) / 2} / n^{1 / 2}$. Also, by Theorem 3.1 , we have $a_{n}^{-1}\left(C^{*}(j)-C^{*}\right) \stackrel{D}{\longrightarrow}$ $\mathcal{N}\left(0, \sigma_{C^{*}}^{2}\right)$, as $n \rightarrow \infty$, where $a_{n}=1 / \sqrt{k_{j}}=2^{j(k(n)) / 2} / k(n)^{1 / 2}$. Relation 3.19), implies that $b_{n}=o\left(a_{n}\right), n \rightarrow \infty$. Indeed, since $k(n)=o(n), n \rightarrow \infty$, we have $2^{j(k(n)) / 2} / n^{1 / 2}=o\left(2^{j(k(n))} / k(n)^{1 / 2}\right) \equiv o\left(a_{n}\right)$, as $n \rightarrow \infty$. This shows that the second term of $b_{n}$ defined above is negligible with respect to $a_{n}$. By Relation (3.19), we also have $k / 2^{j(k)(1+2 \min \{1, \beta\})} \rightarrow 0$, as $k \rightarrow \infty$, or, equivalently $1 / 2^{j(k) \min \{1, \beta\}}=o\left(2^{j(k) / 2} / k^{1 / 2}\right)$, as $k \rightarrow \infty$. Hence, the first term of $b_{n}$ defined above is also of order $o\left(2^{j(k(n)) / 2} / k(n)^{1 / 2}\right) \equiv o\left(a_{n}\right)$, as $n \rightarrow \infty$.

Now, by using the fact that $b_{n}=o\left(a_{n}\right), n \rightarrow \infty$ and the 'Delta-method' applied to the function $f(x, y, z)=$ $2^{x(y-z)}$ and $x_{0}=\alpha, y_{0}=C$ and $z_{0}=C^{*}$ (see also (3.16)), we obtain

$$
a_{n}^{-1}(\hat{\theta}(j)-\theta) \stackrel{D}{\longrightarrow} \partial_{z} f\left(\alpha, C, C^{*}\right) Z \sim \mathcal{N}\left(0, \sigma_{\theta}^{2}\right),
$$

as $n \rightarrow \infty$. Since $\partial_{z} f\left(x_{0}, y_{0}, z_{0}\right)=-\ln (2) \alpha \theta$, we obtain

$$
\sigma_{\theta}^{2}=\left(\partial_{z} f\left(\alpha, C, C^{*}\right)\right)^{2} \sigma_{C^{*}}^{2}=\ln (2)^{2} \theta^{2} \operatorname{Var}\left(\log _{2}(Z)\right),
$$

where $Z$ is a $1-$ Fréchet variable (see Theorem 3.1). Since $\ln (2) \log _{2}(Z)$ has the standard Gumbel distribution, it follows that $\ln (2)^{2} \operatorname{Var}\left(\log _{2}(Z)\right)=\pi^{2} / 6$ (see e.g. (22.31) in Johnson et al. (1994)). This completes the proof of the theorem. 